\title{
High-Energy Cosmic Ray Observations
}

\author{
Heinigerd Rebel ${ }^{*}$ \\ Forschungszentrum Karlsruhe, Institut für Kernphysik, POB 3460, D-76021 Karlsruhe, \\ und Fakultät für Physik und Astronomie der Universität Heidelberg. \\ Email: rebel@ik.fzk.de
}

ABstract: A review is given about current high-energy and ultrahigh-energy cosmic ray studies, in particular with the aspect of the determination of the energy spectrum and elemental composition around the "knee" (at about $4 \cdot 10^{15} \mathrm{eV}$ ), around the "ankle" (at about $5 \cdot 10^{18} \mathrm{eV}$ ) and above. The physical and astrophysical implications of the actual findings are briefly discussed.

Keywords: "̈ịgh-Energy, Āir Shower, Cosmic Ray Ōbservationsi.

\section{Introduction}

As we nowadays know, the energy spectrum of primary cosmic ray particles extends from $1 \mathrm{GeV}$ to above $10^{20} \mathrm{eV}(100 \mathrm{EeV})$, to the highest energies of known individual particles in the Universe. However, we have only a rudimentary understanding of where these particles are coming from, how they are accelerated to such extremely high energies, how they propagate through the interstellar space, and in addition how they do interact with matter. One of the difficulties is that cosmic rays are overwhelmingly charged particles, and the galactic and intergalactic magnetic fields are sufficiently strong to scramble their paths. Perhaps except of the highest energies, cosmic rays have lost all their memory about the location of the emission sources when they eventually arrive the Earth's atmosphere. Hence the only observable quantities, which may give us some information are the energy distribution and the elemental composition of primary cosmic rays. At highest energies above $10^{18} \mathrm{eV}$ the search for possible deviations from isotropic incidence (anisotropies) becomes important. The experimental investigations of the observable features are current topics of contemporary research, especially in energy ranges which exceed the energies provided by man-made accelerators.

The energy spectrum (Fig.1) of primary cosmic rays comprises more than 12 orders of magnitude in the energy scale $(E)$ with a decline of the intensity of more than 30 decades.

${ }^{*}$ Speaker. 


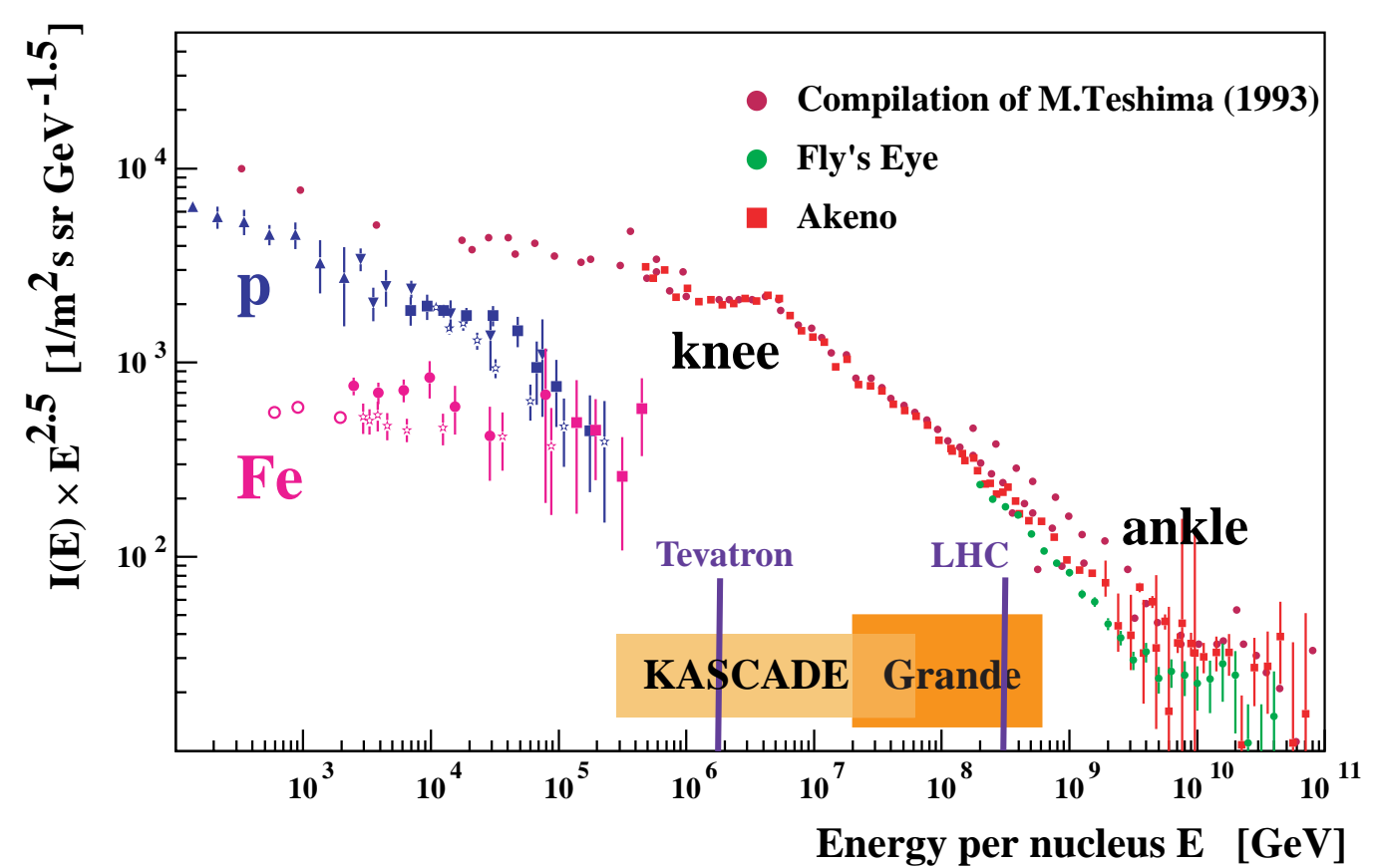

Figure 1: Primary energy spectrum of cosmic rays (note that the flux $I(E)$ is multiplied by $E^{2.5}$ !). The laboratory energies equivalent to the Tevatron collider and LHC c.m. energies are indicated together with the energy range covered by the KASCADE experiment [2].

It follows an overall power-law $\left(\propto E^{-2.7}\right)$, indicating the non-thermal character, with a characteristic distinct change of the spectral index $\gamma$ to $\approx 3.1$ around $10^{15} \mathrm{eV}$, called the "knee". This feature, still not consistently explained, has been discovered 40 years ago by German Kulikov and George Khristiansen of the Moscow State University [1] with studies of the intensity spectrum of Extensive Air Showers (EAS), the so-called shower size, which roughly reflects the primary energy.

The flux of primary cosmic rays falls from 1 particle per $m^{2} \cdot s$ to 1 particle per $\mathrm{km}^{2} \cdot$ century at highest energies. A great deal of interest and current efforts concern the shape of the spectrum in the $E e V$ region, above $10^{18} \mathrm{eV}$, where the spectrum seems to flatten (ankle), and especially around $6 \cdot 10^{19} \mathrm{eV}$ with the theoretically predicted GreisenZatsepin-Kuz'min cut-off (GZK) [3], due to the photo-interaction of protons with the $2.7 \mathrm{~K}$ primordial background radiation. In particular the AGASA experiment in Akeno (Japan) [4] and former (preliminary) observations with the Fly's Eye installation (Utah, USA) [5] seem to show that this limit of the cosmic ray spectrum does probably not exist. This fact is an issue of extreme astrophysical and cosmological relevance (see the discussion of the actual situation in [6]).

Below $10^{14} \mathrm{eV}$ the flux of particles is sufficiently large that individual nuclei can be studied by flying detectors in balloons and satellites. From such direct experiments we know that the majority of particles are nuclei of common elements. Around $1 \mathrm{GeV}$ the abundances are similar to those found in ordinary material of the solar system. Striking exceptions are the abundance of elements like $\mathrm{Li}, \mathrm{Be}$, and $\mathrm{B}$, overabundant since originating 
from spallation of heavier nuclei in the interstellar medium.

At higher energies we rely on observations of Extended Air Showers (EAS), providing indirect information, in the sense that we do not determine directly energy and mass of the primary cosmic particles. The knowledge searched for is inferred from secondary effects, from the lateral and longitudinal development of the particle cascades initiated by the primary cosmic particles in the atmosphere. The following review sketches the current experimental activities in studying high- and ultrahigh-energy cosmic rays (around the "knee", the "ankle" and above) and discusses some methodical aspects of EAS observations and the motivations of the experiments.

\section{Methodical aspects and techniques}

Above $10^{14} \mathrm{eV}$ the techniques used to study cosmic rays exploit the phenomenon of Extensive Air Showers discovered in 1938 independently by Auger [7] and Kohlhörster [8] communicated by timely overlapping publications. Most of the produced particles in the hadron interactions are pions and kaons, which can decay into muons and neutrinos before interacting, thus producing the most penetrating component of atmospheric showers, the muon component. The most intensive component - electrons and photons - originates from the fast decay of neutral pions into photons, which initiate electromagnetic showers, thus distributing the originally high energy over millions of charged particles (Fig.2).

The electromagnetic cascade shows a growth, a maximum and a decay as the energy of the shower is degraded (Fig.3). In contrast the muon cascade grows and maximises, but the decay is only slow as consequence of the relative stability and small cross sections

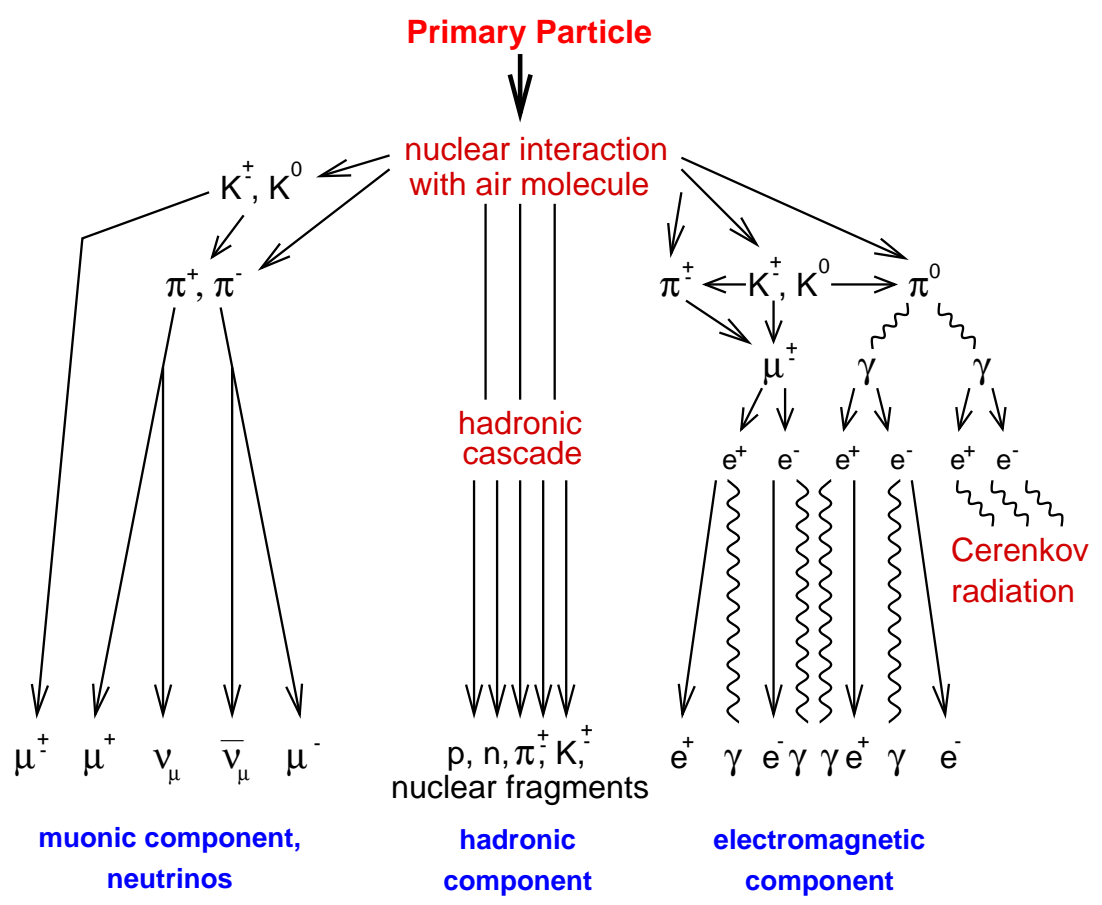

Figure 2: Extensive Air Shower progeny. 


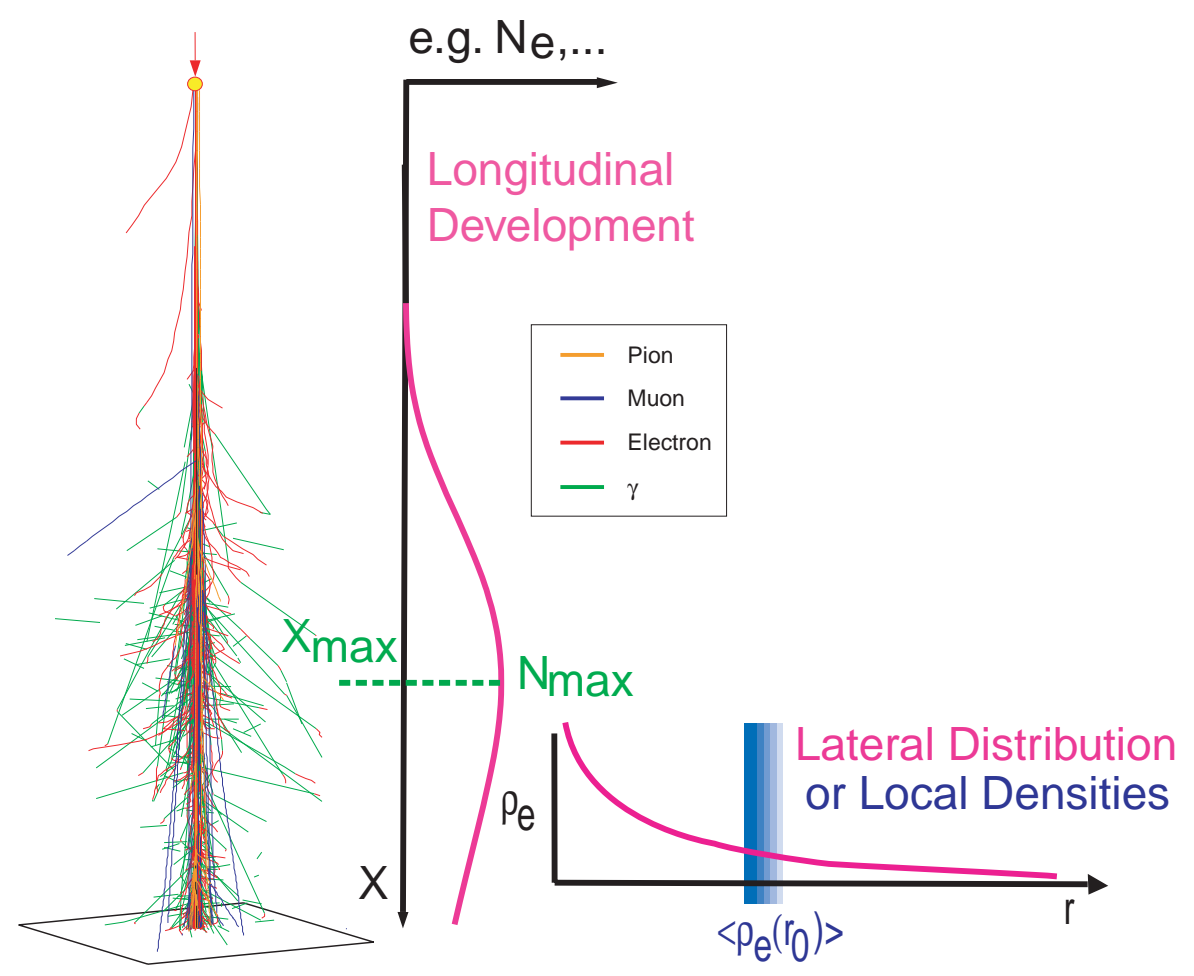

Figure 3: Schematic view of the Extensive Air Shower development.

for ionisation and pair production losses ("penetrating component"). The backbone of an air shower is the hadronic component of nucleons, pions and other particles, which feeds the electromagnetic and muonic components. It is often stated that the hadronic component is well concentrated around the shower axis. Nevertheless due to multiple scattering, neutrons in particular, are distributed also far off the center. There are clear

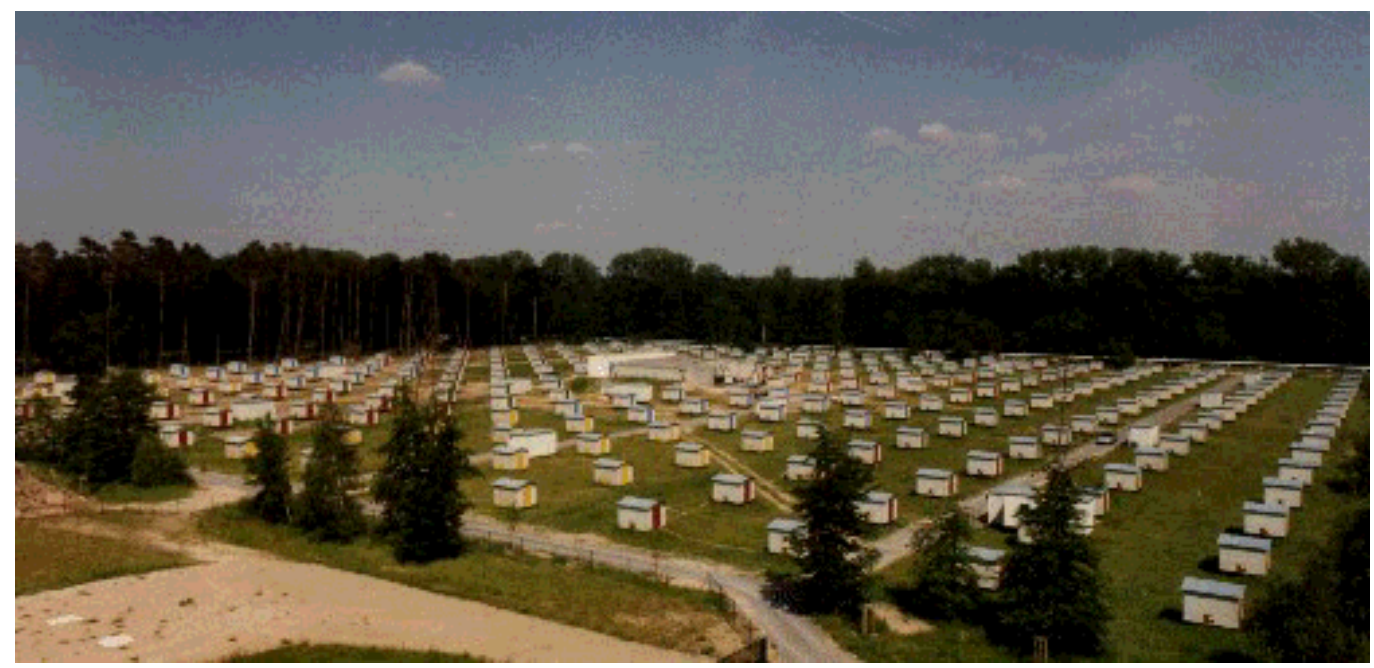

Figure 4: Photo of the KASCADE detector array. 
differences in the longitudinal development of EAS induced by different primaries. The shower development of a heavy ion induced shower starts earlier, is faster and reaches the intensity maximum earlier than it is the case for proton induced showers of the same energy. The gamma-ray induced showers show much less fluctuations and are poor of muons due to the small cross sections of meson production by photons and muon pair production. The electromagnetic component is accompanied by an additional EAS phenomenon, the production of atmospheric Cherenkov light which carries information about the longitudinal shower development, especially about the height $X_{\max }$ of the shower maximum. At high energy the shower can be efficiently observed by the fluorescence light of $N_{2}$ molecules induced by the charged particles in air. Air fluorescence observations are able to reconstruct the shower profile (see section 6). Cherenkov and fluorescence light observations require absence of light background; they need clear moonless nights and have consequently a lower duty cycle for taking data.

Thus, in ground-based experiments with detector arrays, we are hardly in the situation to register the longitudinal development, we observe only the developed status of the air shower cascade at the observation level of the detectors. From the observables there, that means from the total intensity of the various particle components, the lateral and accessible energy distributions, and eventually temporal profiles of the shower disk, we have to deduce the properties of the primary particle.

The intensity and the width of the lateral distributions of the three components are rather different. The muons, for example, extend to several hundred meters as many of them are produced very high in the atmosphere. Therefore, even a small transverse momentum imparted to them in the production process can lead to large lateral distances from
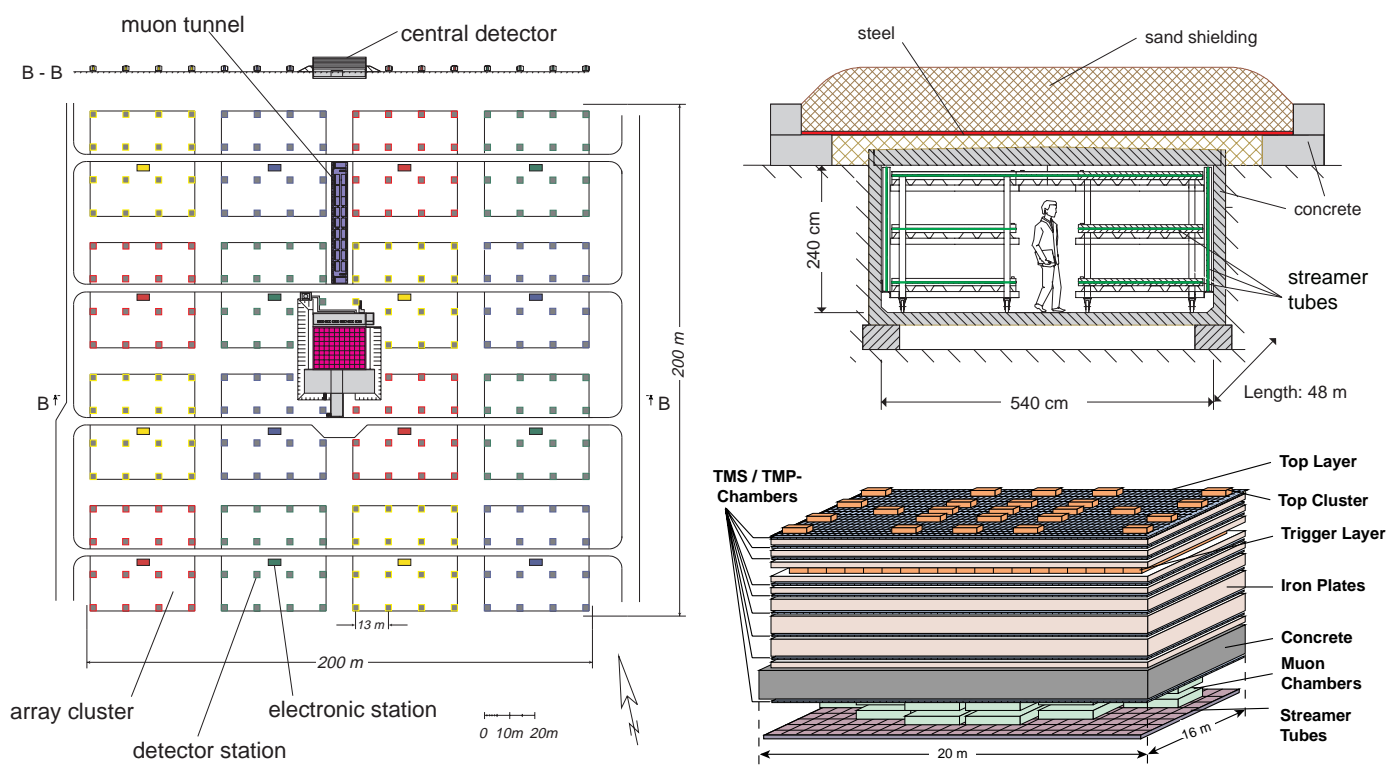

Figure 5: Layout of KASCADE, the muon tracking detector, and the Central Detector System. 


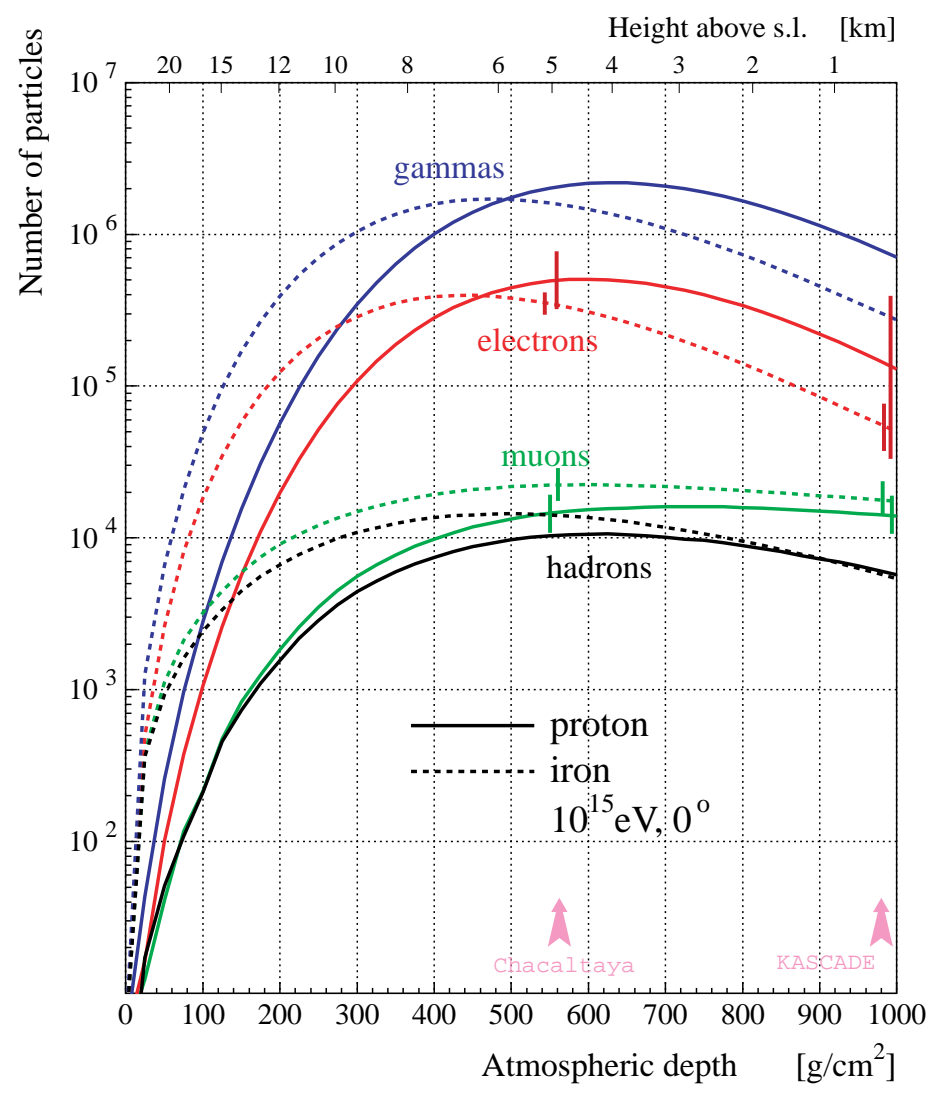

Figure 6: Longitudinal Extensive Air Shower development of the average total intensities (sizes) of different Extensive Air Shower components.

the shower axis at the observation level. In standard EAS experiments the lateral distributions of the particles are sampled by more or less regular arrangements of a large number of detectors which cover only a small fraction of the total area. This sampling is source of instrumental fluctuations which add to the large spread resulting from the inherent statistical fluctuations due to the stochastic shower development in the atmosphere. Just as example the photo (Fig.4) shows the KASCADE detector arrangement [2], installed in Forschungszentrum Karlsruhe. The speciality of KASCADE is, that it measures simultaneously all three main EAS components (including the hadronic component) in an event per event mode.

KASCADE is a multi-component detector array (Fig.5), a field of electron-muon counters and a central detector [9], which is a complex arrangement of several types of detectors, basically an iron sampling calorimeter for hadron measurements and layers of multiwire proportional chambers [10] and limited streamer tubes below, for studies of the higherenergy muon component, and other detectors for special purposes. In addition there is a muon tracking detector (MTD) operated underground with a muon detection threshold of $0.9 \mathrm{GeV}[11]$. The table in Fig.7 compiles various similar cosmic ray detector installations, 
based on different experimental concepts.

Tab.1 compiles some signatures for the mass of the primary cosmic particles. For a more detailed discussion about these observables, their measurements and information content see [12]. The most powerful quantity measured by ground arrays is the correlation between the electron size and the muon content of the EAS. This signature gets obvious from the inspection of the average longitudinal development of the shower sizes (Fig.6).

Due to the shorter interaction length and the smaller energy per nucleon and because of the small attenuation of the muon component, the electromagnetic component of a heavyparticle induced shower develops faster in average, and the shower carries more muons than a proton induced shower of the same energy is doing. This feature is the basis of the electron-muon correlation method and its variants.

Tab.1. $\quad$ Signatures of the cosmic ray mass composition from EAS observations.

- Shower size $N_{e}$ - Muon Content $N_{\mu}$ - Correlation. Basis: Weak dependence of the multiplicity $\mathrm{n}$ of the secondaries from energy of the interacting nucleon

$$
\begin{aligned}
& n_{\text {prot }}(E) \propto \ln E \\
& n_{A}(E) \propto A \ln (E / A)
\end{aligned}
$$

combined with the faster longitudinal development of heavy primaries induced showers.

- Structure of the Energy and Lateral Distributions of Muons and Hadrons in the Shower Core. Basis: Smaller deflection angles and a harder energy spectrum of the secondaries in proton induced showers as compared to heavy ion induced showers of the same primary energy.

- Distributions of the Relative Arrival Times and Angles of Incidence of the Muon Component. Basis: Mapping differences of the longitudinal development and of the mean production height of the muon component.

- Underground Observation of the Muon Lateral Distribution: Muon Bundles. Basis: Differences of the spectra and lateral distributions of highenergy EAS muons.

- Observation of the Pulse Shape and Lateral Distribution of the Air Cherenkov Light. Basis: Mapping of the longitudinal EAS profile (depth $X_{\max }$ of the EAS maximum) by the arrival times and lateral distribution of the Cherenkov photons.

- Observation of the $N_{2}$ Fluorescence Light. Basis: Tracking of the shower profile and observation of $X_{\max }$. 


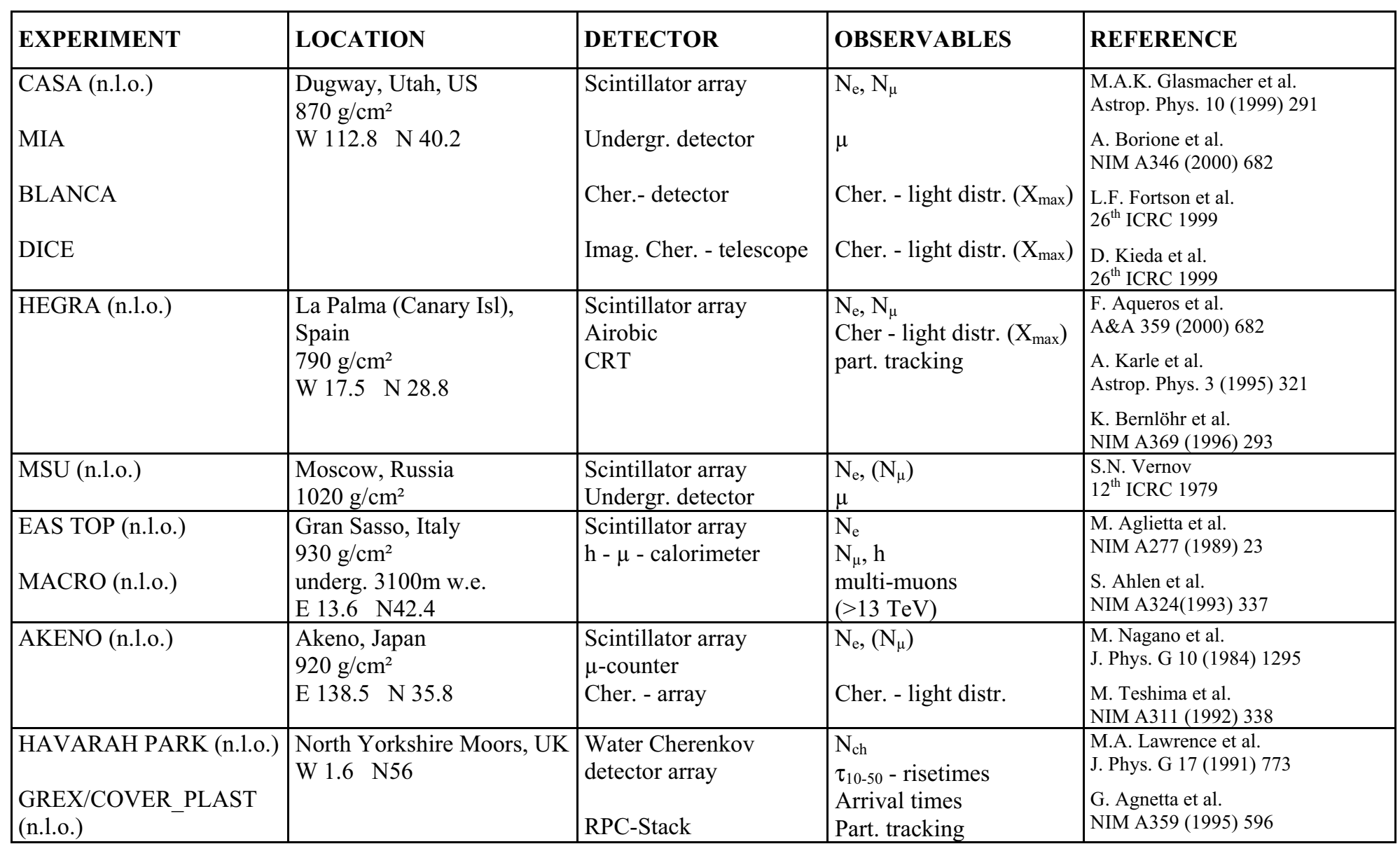




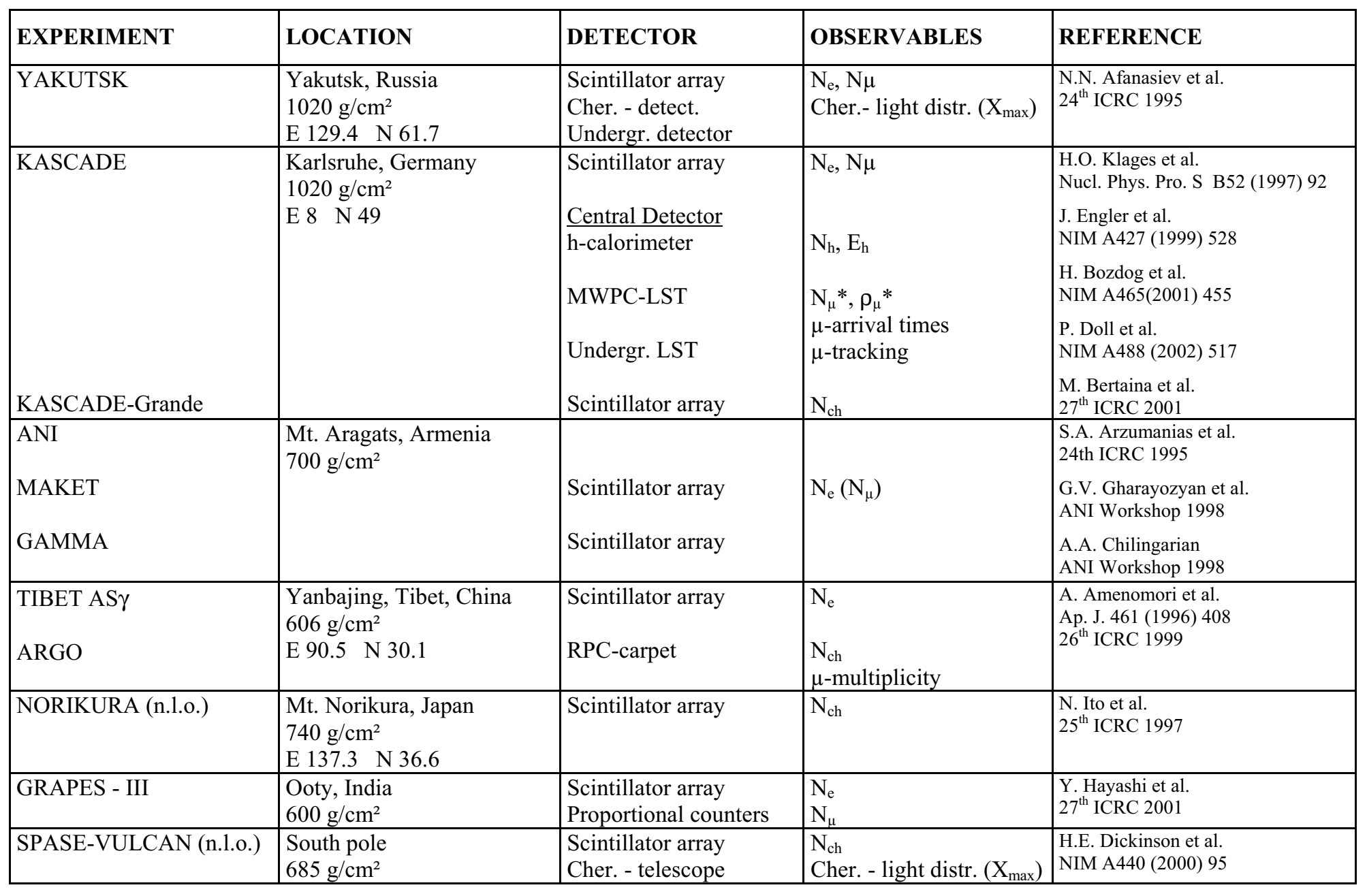




\begin{tabular}{|c|c|c|c|c|}
\hline EXPERIMENT & LOCATION & DETECTOR & OBSERVABLES & REFERENCE \\
\hline Tien-Shan & $\begin{array}{l}\text { B. Alma - Ata } \\
690 \mathrm{~g} / \mathrm{cm}^{2}\end{array}$ & Cher. - det. array & Cher. - light distr. & \begin{tabular}{|l} 
R.A. Antonov et al. \\
$26^{\text {th }}$ ICRC 1999
\end{tabular} \\
\hline $\begin{array}{l}\text { L3 - COSMICS (n.1.o.) } \\
\text { COSMO-ALEPH (n.l.o.) }\end{array}$ & $\begin{array}{l}\text { LEP - CERN, Switzerland } \\
1000 \mathrm{~g} / \mathrm{cm}^{2}\end{array}$ & $\mu$ - Spektr. & $\begin{array}{l}\mathrm{N}_{\mathrm{e}}, \mathrm{N}_{\mu} \\
\text { multi-muons }\end{array}$ & $\begin{array}{l}\text { B. Adeva et al. } \\
\text { NIM A289 (1990) } 35 \\
\text { O.Adriani et al. } \\
\text { NIM A488 (2002) } 209\end{array}$ \\
\hline TUNKA 13 & \begin{tabular}{|l|} 
Russia \\
$950 \mathrm{~g} / \mathrm{cm}^{2}$ \\
$\mathrm{E} 103 \quad \mathrm{~N} 51.5$ \\
\end{tabular} & Cher. - detector & Cher. - light distr. $\left(\mathrm{X}_{\max }\right)$ & $\begin{array}{l}\text { O.A. Green et al. } \\
\text { Nucl. Phys. B (Proc. Suppl.) 75A } \\
\text { (1959) } 299\end{array}$ \\
\hline $\begin{array}{l}\text { BAKSAN ANDYRCHY } \\
\text { BUST }\end{array}$ & $\begin{array}{l} \\
833 \mathrm{~g} / \mathrm{cm}^{2} \\
\mathrm{E} 42.7 \quad \mathrm{~N} 43.4\end{array}$ & $\begin{array}{l}\text { Scintillator array } \\
\text { Undergr. telescope } \\
\text { array }\end{array}$ & $\begin{array}{l}\mathrm{N}_{\mathrm{ch}} \\
\text { multi-muons }\end{array}$ & \begin{tabular}{|l} 
E.N. Alexejev et al. \\
$23^{\text {th }}$ ICRC 1993 \\
E.N. Alexejev et al. \\
ICRC 1979 \\
\end{tabular} \\
\hline $\begin{array}{l}\text { CHACALTAYA } \\
\text { BASJE } \\
\text { PAMIR }\end{array}$ & $\begin{array}{l}\text { Mt. Chacaltaya, Bolivia } \\
530 \mathrm{~g} / \mathrm{cm}^{2} \\
\text { W } 68.2 \text { S } 16.4 \\
\text { Tadjikistan, Pamir plateau } \\
594 \mathrm{~g} / \mathrm{cm}^{2}\end{array}$ & $\begin{array}{l}\text { Emulsion ch. } \\
\text { Scintillator array } \\
\text { Emulsion.ch }\end{array}$ & $\mathrm{N}_{\mathrm{e}}$, hadrons & $\begin{array}{l}\text { Y. Shirasaki et al. } \\
\text { Astrop. Phys. } 15 \text { (2001) } 357\end{array}$ \\
\hline
\end{tabular}


Adjusted to the particular detector capabilities various different energy estimators have been developed. The KASCADE experiment uses for an approximate energy identification the truncated muon number $N_{\mu}^{t r}$, i.e. the muon intensity integrated between $40 \mathrm{~m}$ and $200 \mathrm{~m}$ distance from the shower axis. Simulation studies show that this estimator is nearly independent on the mass of the primary in the energy range covered by KASCADE. The estimate can be improved by a combination $a_{e} \log N_{e}+a_{\mu} \log N_{\mu}$. The former $\left(1 \mathrm{~km}^{2}\right)$ Akeno array [13] relates $N_{e}$ to the primary energy by some experimental information about $N_{\max }$. Larger surface detector arrays like Haverah Park [14], AGASA [4] and Yakutsk [15] derive energy estimators from the charge particle density $S(600)$ measured in $600 \mathrm{~m}$ distance from the EAS axis (see section 7). The total Cherenkov and fluorescence light produced in the atmosphere by the EAS is used for the energy estimate (see section 6), when these EAS components are observed.

However the stochastic character of the huge number of cascading interactions in the shower development implies considerable fluctuations (Fig.8) of the experimentally observed EAS parameters, obscuring the properties of the original particle. These inherent (unavoidable) fluctuations establish an important and intriguing difficulty of any EAS analysis. They impose limits to approximations by parameterisations, and they need adequate response by the analysis methods.

The general scheme of inference in a modern EAS experiment is displayed in the Fig.9, indicating also the involved difficulties.

The identification of differences in EAS which result from differences in mass of the primary particle requires a modeling of the shower development in the atmosphere. For that Monte-Carlo programs of the EAS development like the Karlsruhe CORSIKA [16] program have been developed. CORSIKA is under continuous modification and improvement. A prerequisite for the Monte-Carlo procedures is the knowledge about particle production in high-energy hadronic interactions: The cross sections, the multiplicity, the rapidity and $p_{t}$-distributions and the inelasticity of the multiparticle production in the hadron collisions. Since the energy region of our interest exceeds the particle energies provided with man made accelerators, we rely on model descriptions which extend the present knowledge to a terra incognita, on basis of more or less detailed theoretical approaches of phenomenological nature and with QCD inspired ideas (see e.g. [17,18]). The CORSIKA code includes various models, presently en vogue as options, and in fact, the model dependence is an obviously pronounced feature in the comparisons with the experimental data.

The further processing is to compare real data with pseudo experimental data on equal level, including the detector response and expressed by various reconstructed shower variables: Intensity, the lateral, arrival time and eventually energy distributions of the various EAS components. One type of analysis is based on comparisons by use of plausible a-priori parameterisations of the considered averaged distributions, with introducing some constraints by analytical expressions and eventually neglecting the fluctuations.

For the need of more efforts for the analysis of the correlated distributions with avoiding any bias of constraining parameterisations, adequate pattern recognition methods, involv- 


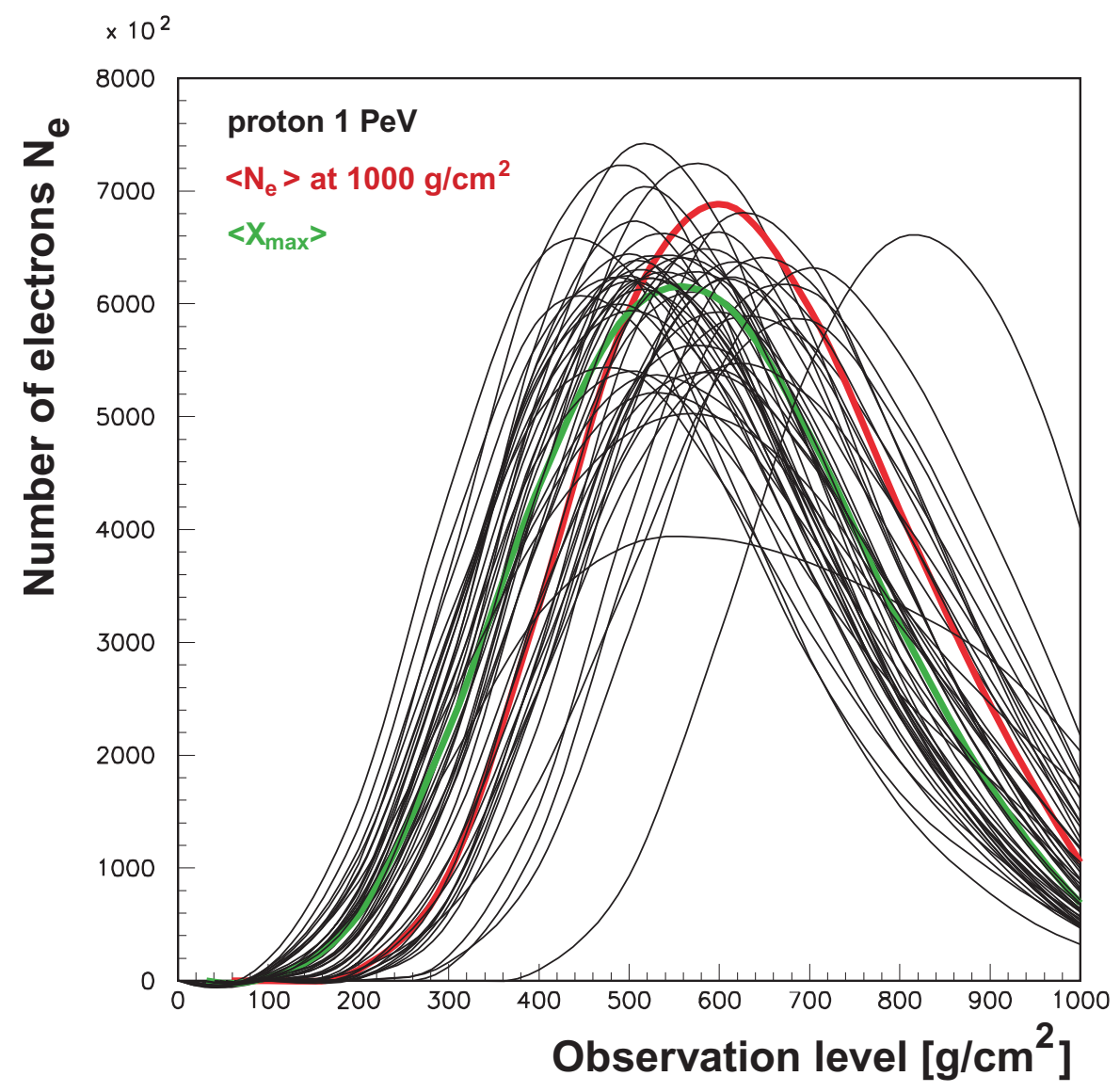

Figure 8: Fluctuations of the development of 50 (simulated) proton induced 1 PeV Extensive Air Showers of vertical incidence. An important reason is the fluctuation of the starting depths.

ing neural networks and Bayesian decision making, have been developed. Applying such non-parametric techniques in an event-by-event mode for each particular case, i.e. for a particular set of selected EAS variables, for a chosen number of mass groups and for a specific hadronic interaction model generating the reference pattern, matrices for true and misclassification are obtained. From that also measures for the confidence and errors can be constructed [19].

For the comparison of the measured observables with the pseudo data we have to realise: None of the observables is strictly dependent only on the mass of the primary, or dependent only of the energy. Since we are investigating an a-priori unknown spectral distribution accompanied by another a-priori unknown variation of the elemental composition (or vice versa), there is always an intriguing feedback of the estimates of both. Therefore multivariate analyses, correlating the observations of different EAS variables are finally strongly required, and the inference from only one single EAS component has proven to be often misleading.

It should be also emphasised: A multi-detector experiment observing simultaneously all major EAS components with many observables provides some good possibilities to test 
KASCADE-Experiment
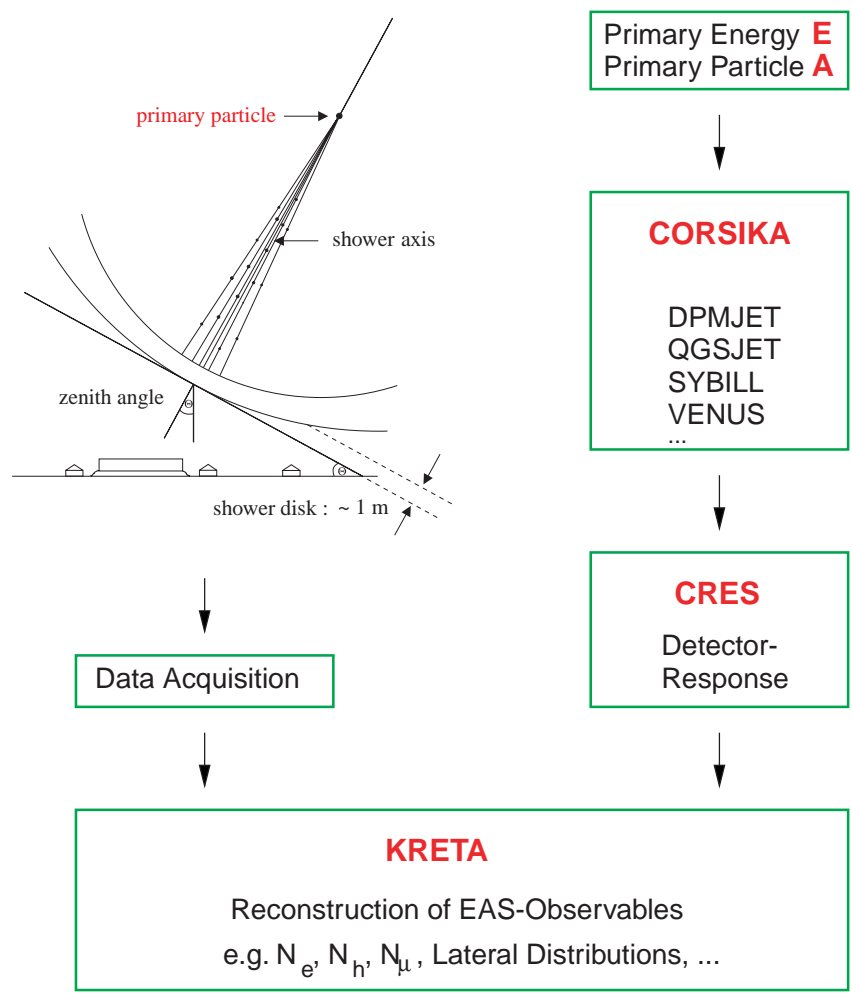

Reconstruction of EAS-Observables

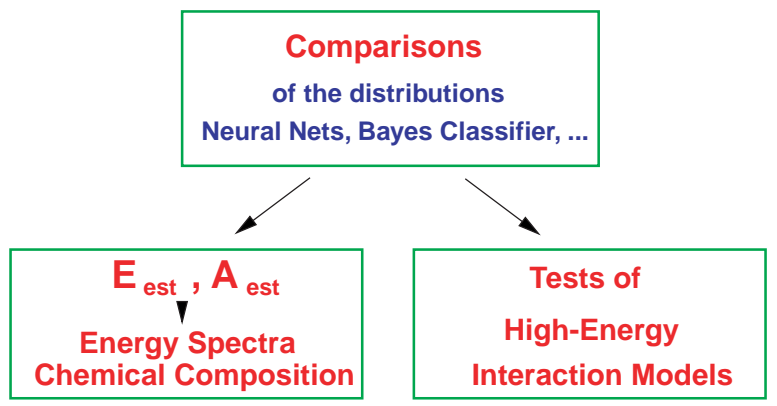

Figure 9: General scheme of the analysis of Extensive Air Showers observations.

the hadronic interaction models and to specify the most consistent one [20,21]. Actually this is another facet of the high energy cosmic ray observations which cannot be disentangled from the astrophysical implications of EAS observations: Looking for information on the hadronic interaction up to energy regions which may be never get explored by artificial accelerators.

\section{The knee}

It is currently believed that cosmic rays are accelerated in a process called diffusive shock acceleration. Suitable astrophysical shocks occur, for example, as result of supernova ex- 
plosions, and the particles of the interstellar medium gain energy as they are repeatedly overtaken by the expanding shock wave. Such mechanisms (see e.g. [22,23]) lead in fact to a power law spectrum with the maximum energy of about $Z \cdot 10^{15} \mathrm{eV}$, what roughly agrees with observed steepening (though the theoretically predicted spectral index proves to be lower than actually observed). Accidentally an explanation of the knee by the (rigidity dependent) propagation of the cosmic particles in the galactic magnetic field (Leakage Box Model) [24] leads also to a change of the spectral index due to the decreasing containment and rising leakage with the rigidity of the (light) particles. An interesting hypothesis about origin of cosmic rays around the knee has been propagated by Erlykin and Wolfendale [25], predicting a modulation in the energy spectrum, i.e. structures (wiggles) due to the various mass production spectra of a single supernova source, localised only a few hundred light years away from our solar system. Alternatively to such astrophysical reasons a sudden change of the character of the hadronic interaction at higher energies has been proposed as explanation of the knee [26].

In order to constrain the various models and conjectures a better knowledge of the shape of energy spectrum and elemental composition around the knee is quite important. In particular, all approaches accounting for the origin and acceleration mechanism, imply specific variations of the elemental composition of primary cosmic rays, sometimes in a very detailed manner. These are the issues addressed by the KASCADE experiment set up on the site of Forschungszentrum Karlsruhe and by other current experiments (see Tab.1).

The concept of the KASCADE experiment with a multi-component detector array is to measure a large number of EAS observables for each individual event with high accuracy. For this aim the detector has been designed. Specific EAS quantities accessible, in addition to the shower size $N_{e}$ and the truncated muon number $N_{\mu}^{t r}$, are the number of hadrons $N_{h}^{100}$ with energies larger than $100 \mathrm{GeV}$, the energy sum $\Sigma E_{h}$ of the hadrons, the energy of the most energetic hadron $E_{\text {hmax }}$ [27], the number $N_{\mu}^{*}$ of muons with energies larger than $2.4 \mathrm{GeV}$ (with the spatial pattern in the shower core) [28]) and others like some quantities relating to longitudinal EAS profile via the temporal structure of the shower disk (muon arrival time distributions [29]) and angles-of-incidence distributions of muons [30].

In the following predominantly results which are deduced by non-parametric analysis procedures are shown.

In these analysing techniques vectors $\mathbf{v}=\left(N_{e}, N_{\mu}^{t r}, N_{\mu}^{*}, N_{h}, \ldots ..\right)$ of observables for each observed event are considered. As first step reference patterns i.e. likelihood distributions $P\left(\mathbf{v} / \omega_{i}\right)$ are generated for the observable vector by invoking predictions from Monte-Carlo simulations for the distributions of different kinds of primaries. Such likelihood functions $P\left(\mathbf{v} / \omega_{i}\right)$ are conditional probability density distributions for observing the multidimensional event being associated to a particular class $i=p, C, F e, \ldots$.

The non-parametric techniques do not parameterise such distributions with a-priori adopted functional forms but take them as a distributions of Bayes classifiers (or of the weights of neural networks). For the comparison of the measured vectors $\mathbf{v}$ with the reference patterns, the Bayes theorem is invoked to transform the likelihood $P\left(\mathbf{v} / \omega_{i}\right)$ that the event $\mathbf{v}$ is associated to the class $\omega_{i}$ to the searched (a-posteriori) probability $P\left(\omega_{i} / \mathbf{v}\right)$ that a certain class $\omega_{i}$ can be assigned to the measured event $\mathbf{v}$. 


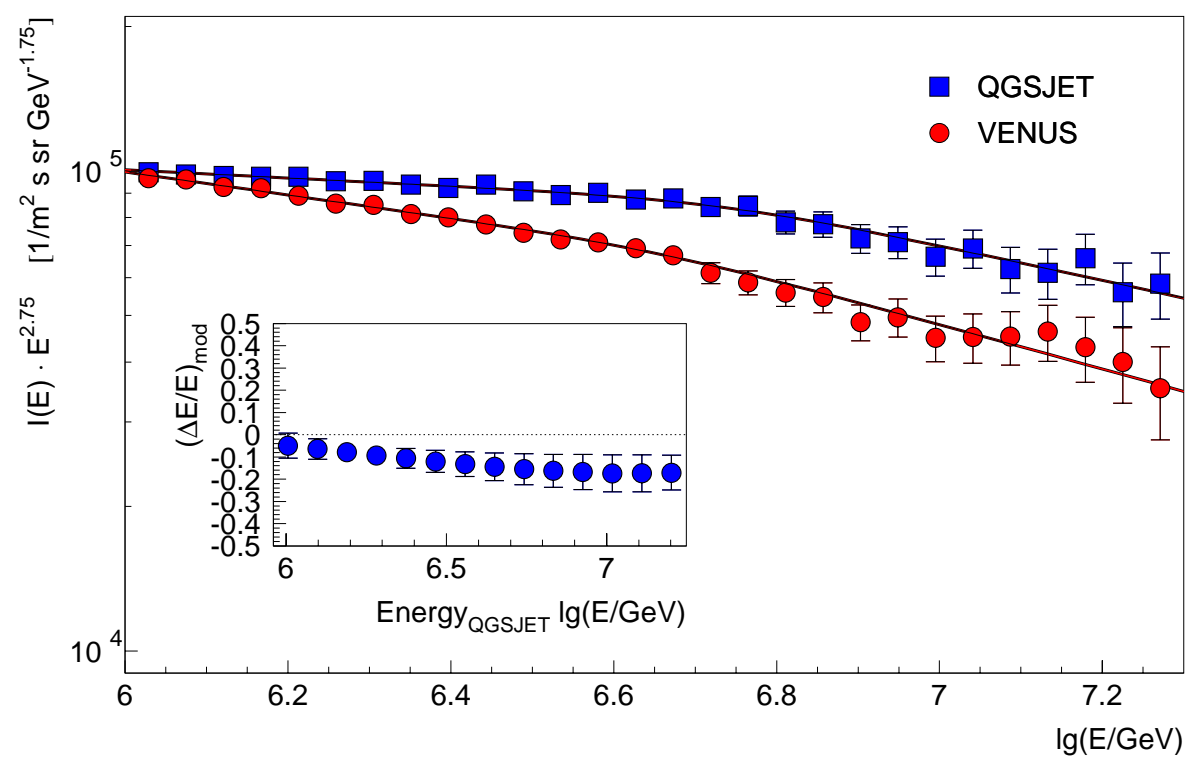

Figure 10: All particle spectrum resulting from a non-parametric analysis of KASCADE data [20].

$$
P\left(\omega_{i} / \mathbf{v}\right)=P_{\omega_{i}} / P_{\text {all }} \cdot P\left(\mathbf{v} / \omega_{i}\right)
$$

Thereby some a-priori knowledge of the relative abundance $P_{\omega_{i}} / P_{\text {all }}$ of the classes enters in the inference procedure. The consistency of the initial assumptions $P_{\omega_{i}} / P_{\text {all }}$ (a-priori probabilities) is just the subject to get improved by the iterative application of the procedure.

Fig.10 presents two solutions of the energy spectrum [20] with the same data and the same analysis procedures, but based on reference patterns from different hadronic

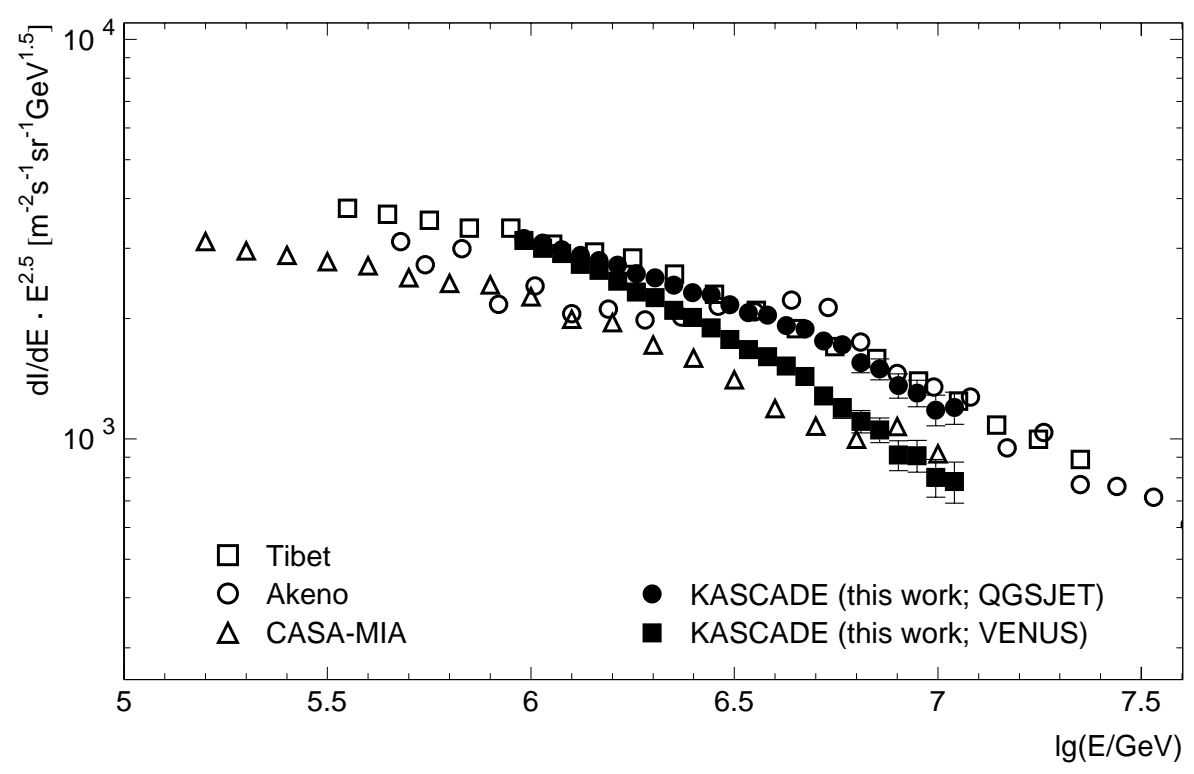

Figure 11: Comparison with other experiments [34,35,36]. 


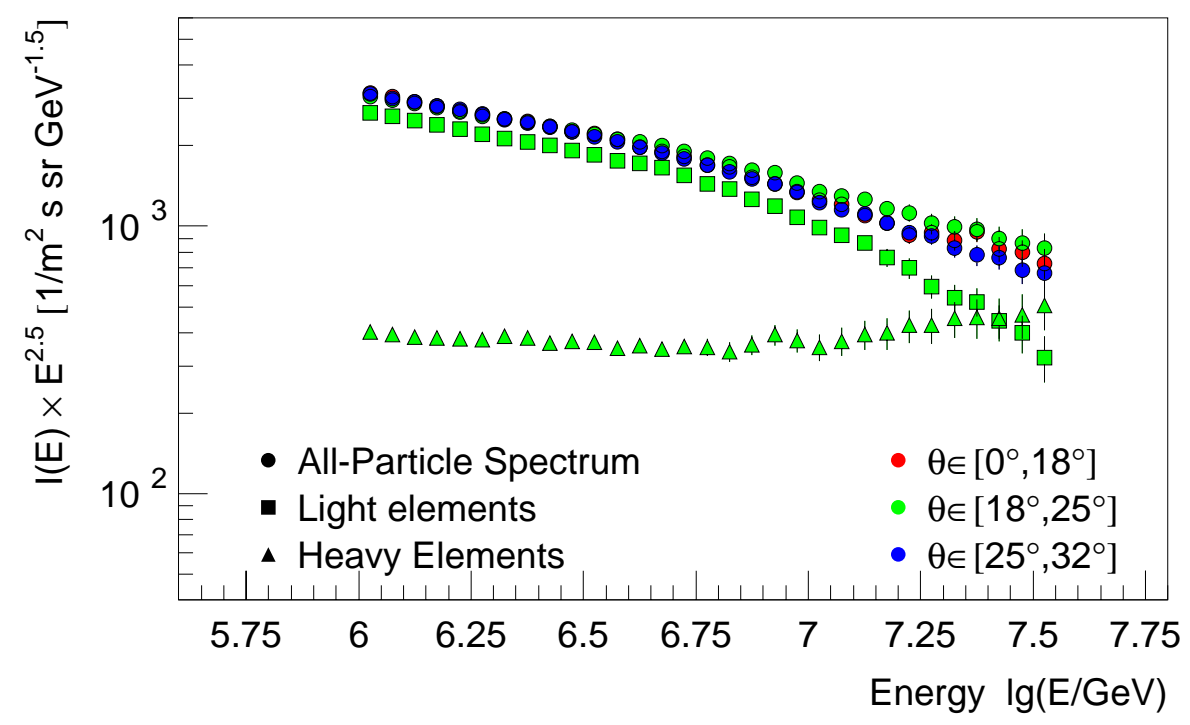

Figure 12: Energy spectra of various mass groups [37].

interaction models, QGSJET [31] and VENUS [32], respectively. These results emphasise and illustrate clearly the present limits due to the unavoidable model dependence of any EAS analysis. From a study [33] of the various hadronic observables the QGSJET model seems to be preferable.

The results (Fig.11) should be seen under various aspects of current controversial discussions: Is there an abrupt break in the spectrum at the knee (as the Akeno observations claim [34] and where it is located? Or is the change of the spectral index rather smooth as obviously observed in the high altitude Tibet array [35] (4300 $\mathrm{m}$ a.s.l.)?
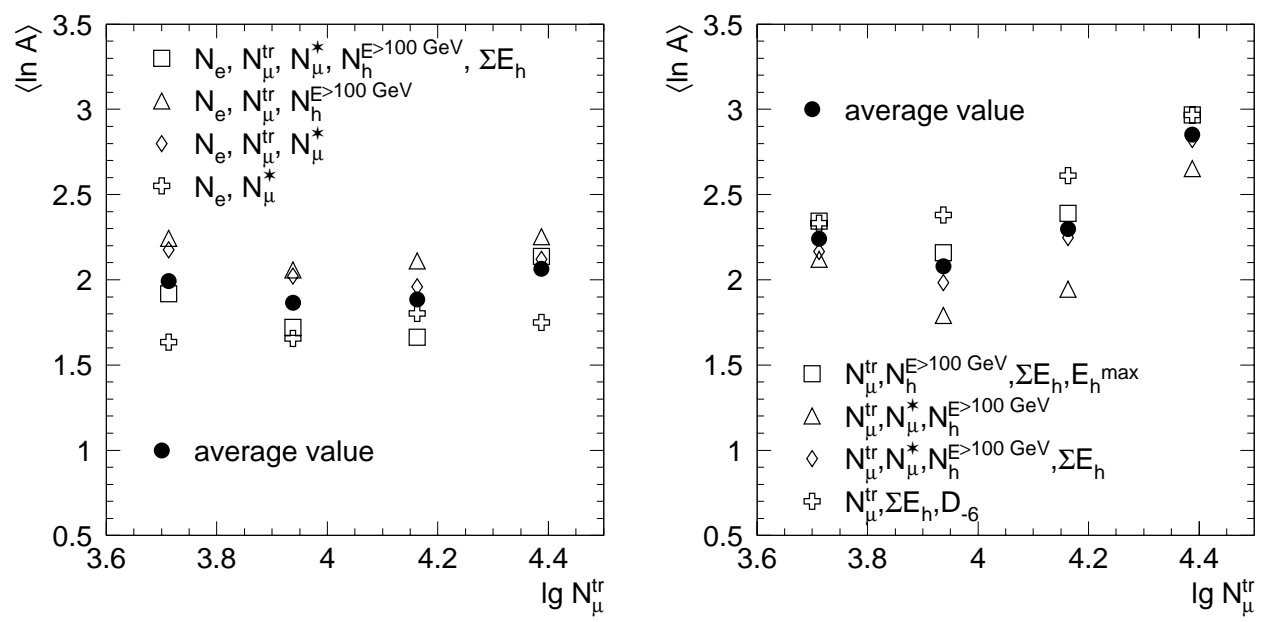

Figure 13: Variation of the mean mass [20]. 

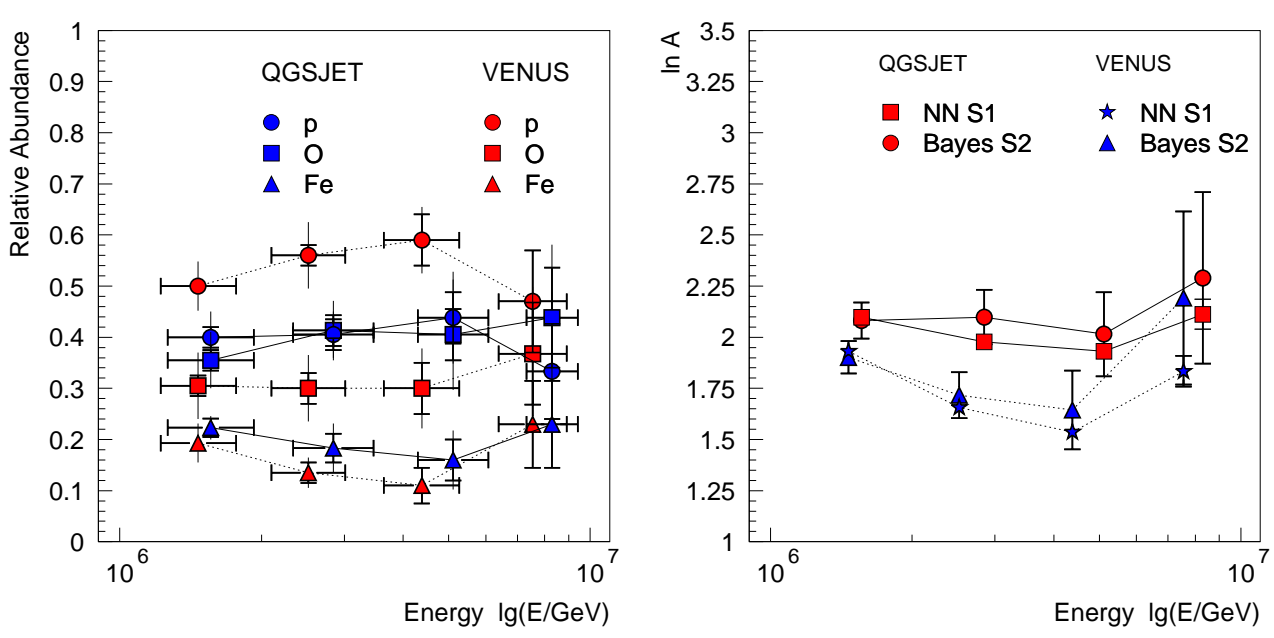

Figure 14: The relative abundances of the three mass classes $p, O, F e$ and the mean logarithmic mass $<\ln A>$ vs. $\log N_{\mu}^{t r}$ [20].

The resulting mass composition may be characterised by the energy spectra of various mass groups. Fig.12 displays the spectra for the light and heavy elements, and the allparticle spectrum is shown for different angular ranges of incidence.

The interesting result is: The knee i.e. the change of the spectral index in the allparticle spectrum is only due to the light component. This feature implies the question: Where is the knee of the heavy component? There has to be a knee to agree with the total flux.

In Fig. 13 the variation of the chemical composition shown by the mean logarithmic mass $<\ln A>$ vs. the energy identifier $N_{\mu}^{t r}$ (the knee is at $\log \left(N_{\mu}^{t r}\right) \approx 4.1$ ), resulting from different sets of observables (right: note that the sets do not include the observable $N_{e}$ ). Fig.14 compares the results obtained with two different hadronic interaction models QGSJET and VENUS for the case of three mass groups (and considering only the $N_{e}-N_{\mu}^{t r}$ correlation). We recognise the general tendency that the lighter composition before the knee gets heavier beyond. The QGSJET model leads generally to a heavier composition. The reconstructed mean mass value depends also of the number of mass classes, in addition to the particular observable set (adding $N_{e}$ or not e.g.). The feature, that scrutinising different observables combinations does not lead to identical results, reflects the internal inconsistency of the actual model providing the reference pattern of the analysis.

The shown results have been recently improved in statistical accuracy [37], and they are in agreement with the outcome of other (parametric) analysis methods ("unfolding techniques") applied to the data. The current status (27th ICRC, Hamburg, Germany, 2001) of the KASCADE approach is represented by Fig.15 (from [38]). 


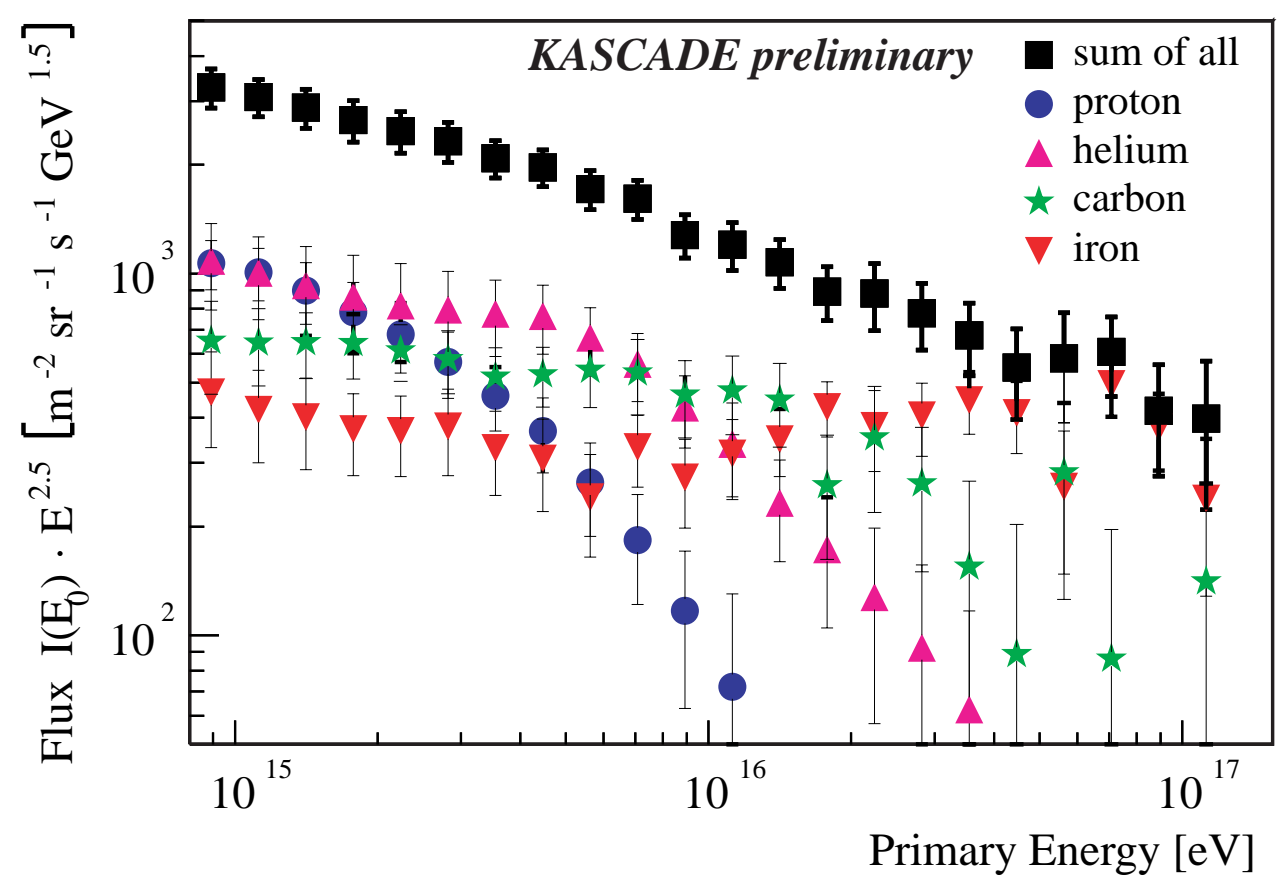

Figure 15: Energy spectra of four primary mass groups (preliminary) as resulting from an unfolding procedure (see [38]). The error bars represent the statistical uncertainties dominated by the limited number of Monte-Carlo simulations.

\begin{tabular}{l}
\hline \hline Tab.2. Focal points of the cosmic ray research in the knee region. \\
\hline \hline - The detailed shape of the energy spectrum around the knee. Smooth \\
or a sharp change of the spectral index? With fine structures? Variation of \\
the elemental composition Knee positions of different elemental components: \\
Scaling with Z or A? \\
- Test of astrophysical models of cosmic ray sources and acceleration \\
mechanisms. \\
- Hadronic Interactions in the "terra incognita" $>10^{15} \mathrm{eV}$ and unex- \\
plored phase space regions.
\end{tabular}

Non-parametric procedures indicate the way, how the data can be consistently analysed, on event-by-event basis with minimum bias and with explorations of the particular sensitivities and uncertainties, arising from the model dependence, e.g. . However, there is still a number of systematic influences which need clarification, though there is no doubt about the trend: Beyond the knee the composition gets heavier and the knee originates from the light component.

The question: "Where is the knee of the iron component?" has prompted an extension of KASCADE to KASCADE-Grande [39] $\left(700 \cdot 700 \mathrm{~m}^{2}\right)$, covering the area of the 


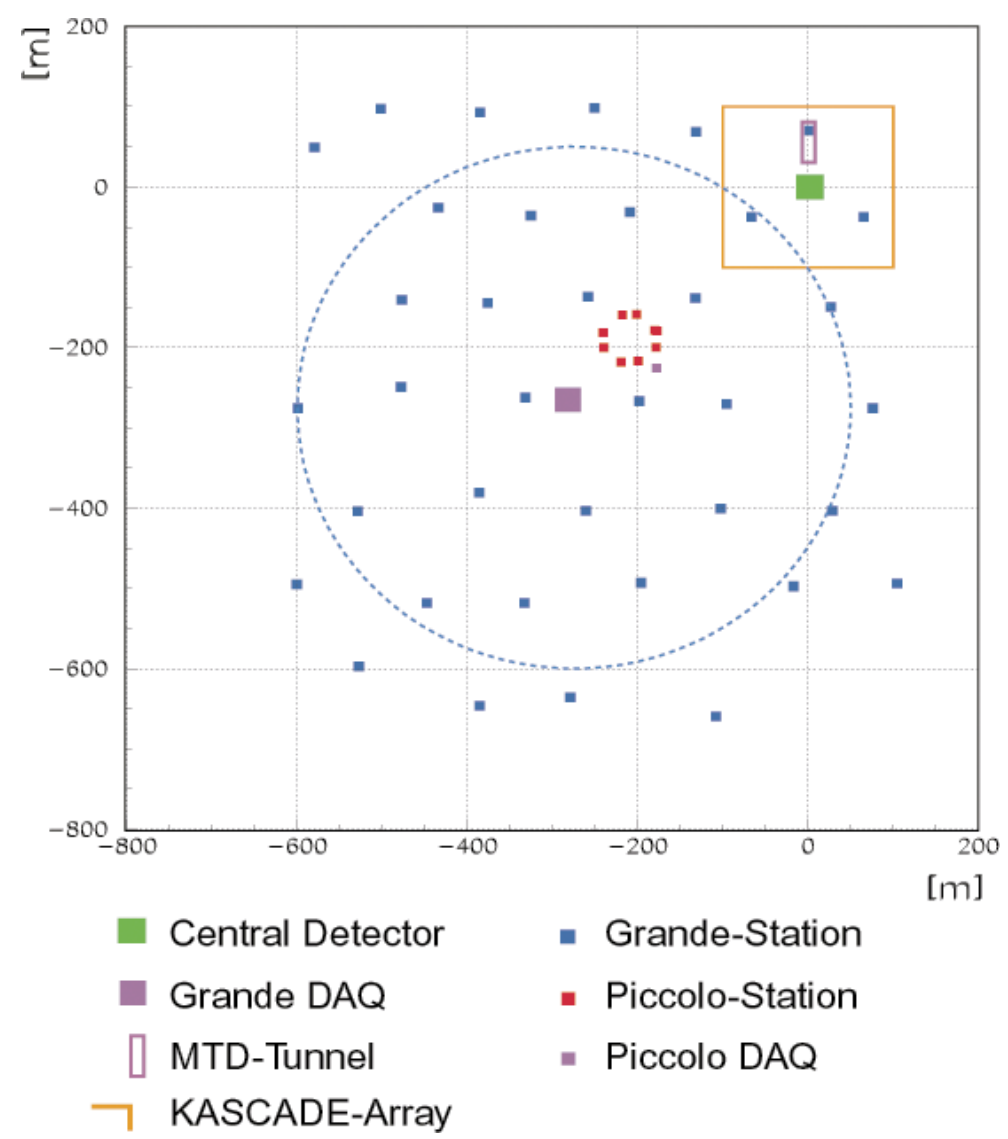

Figure 16: Layout of KASCADE-Grande, including the original KASCADE array (with all facilities) and a new trigger array Piccolo [39].

Forschungszentrum Karlsruhe with the scintillation detector stations from the EAS-TOP experiment (Fig.16). With this array EAS observations up to $10^{18} \mathrm{eV}(1 \mathrm{EeV})$ are planned, starting with full data taking in 2003.

The KASCADE results are corroborated by the trend of results of other experiments (see e.g. [36]). As example Fig.17 displays a compilation of measurements of the height $X_{\max }$ of the EAS maximum resulting from various experiments with Cherenkov light observation. By this technique the lateral distribution of the Cherenkov light is registered on ground. As justified by Monte-Carlo simulations, the lateral distribution (slope and relative intensities at certain distances) can be related to the distance of the shower maximum $[40,41]$.

Strictly the conversion of the shape of the lateral distribution of the Cherenkov light into $X_{\max }$ is not free from some bias of model predictions. The analysis is a typical single parameter study of an observable whose mean value may be interpreted in terms of an adopted composition model or of the mean mass of the primary deduced as astrophysically interesting quantity.

One has to realise that the interpretation of $X_{\max }$ in terms of a composition is model dependent, as the theoretically predicted variation displays in Fig.17, calculated for two 


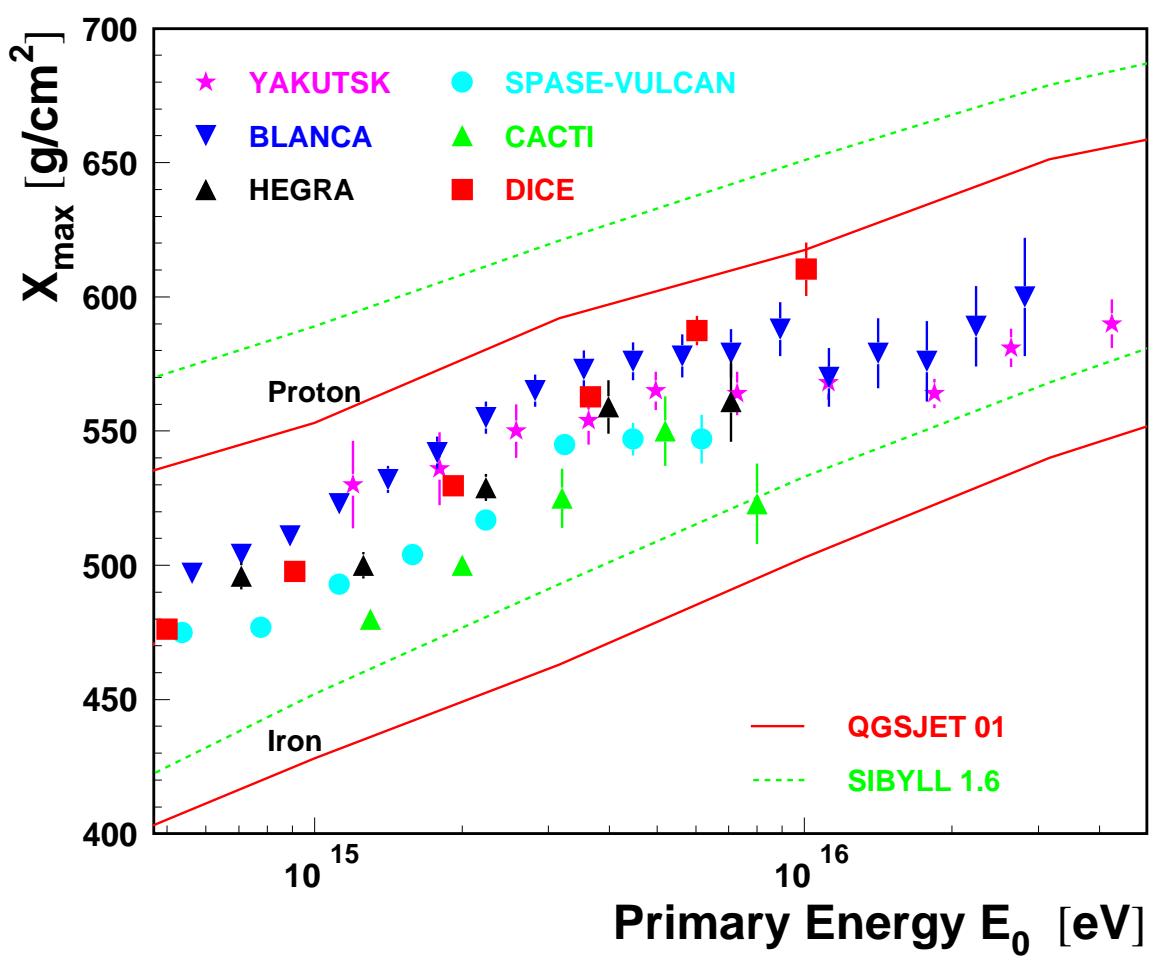

Figure 17: Compilation: Variation of the height of the EAS maximum $X_{\max }$ from various experiments: BLANCA [42], CACTI [43], DICE [44], HEGRA [45], SPASE VULCAN [46], YAKUTSK $\left[4^{7}\right]$.

different interaction models (see also the illustration in [42]).

With summarising the aims of future cosmic ray research around the knee (Tab.2) we characterise the present status by the obvious dilemma, that the analyses of the measured data are limited by a distinct dependence on the adopted high-energy interaction model. Though, in contrast to other current experiments, the KASCADE experiment is able to specify the inherent model dependence, thanks to the large number of observables studied simultaneously in an event-by-event mode, any substantial progress needs an improved knowledge of the interaction model.

\section{From where may we get improved information about the hadronic interaction?}

Microscopic hadronic interaction models used as generators of EAS Monte-Carlo simulations by programs like CORSIKA, MOCCA [48], or AIRES [49] e.g. are based on partonparton interactions and approaches, inspired by QCD, considering the lowest-order interaction graphs of the elementary constituents of hadrons. However, there are not yet exact ways to calculate the bulk of soft processes since for small momentum transfer the coupling constant $\alpha_{s}$ of the strong interaction is so large that perturbative QCD fails. Thus we have to rely on phenomenological models which incorporate concepts of scattering theory. A class of successful models are based on the Gribov-Regge theory which finally leads to 
descriptions of colour exchange and re-arrangements of the quarks by string formation. The various string models differ by the types quark lines included. The momenta of the participating partons are generated along the structure functions. The models are also different in the technical procedures, how they incorporate hard processes, which can be calculated by perturbative QCD. With increasing energy hard and semihard parton collisions get important. Thus the string models like SYBILL [50], QGSJET [31], VENUS [32], DPMJET [51] (and others and in different updated versions), which are specifically en vogue as generators of EAS Monte-Carlo simulations, are based on the Gribov-Regge theory. They describe soft particle interactions by exchange of one or multiple Pomerons. Inelastic reactions are simulated by cutting Pomerons, finally producing two colour strings per Pomeron which subsequently fragment into colour-neutral hadrons. The differences between the models arise from some details in the treatment and fragmentation of strings. An important difference is that QGSJET and DPMJET are both able to treat hard processes, whereas VENUS does not do so. VENUS on the other hand allows for secondary interactions of strings which are close to each other in space and time. When we realise that the present experimental reach ends at some $10^{15} \mathrm{eV}$ (equivalent laboratory energy of the Tevatron collider) and that such models have to be extended to ultrahigh energies over several decades, it is a question of urgent interest how the theoretical developments can be guided and controlled by experimental findings. Thereby one has to emphasize that the efforts have to be predominantly focused to the forward i.e. diffraction region with low momentum transfer of the hadronic collisions, since this part of the kinematical range drives the development of the air showers. With this view the schedules and experimental plans of current and forthcoming accelerator experiments have been recently discussed in a workshop organised in Forschungszentrum Karlsruhe: "NEEDS from Accelerator Experiments for Understanding High Energy Extensive Air Showers" [52], developing a list of desired information from accelerator experiments that could be used for constructing more accurate and realistic hadronic interaction models.

However, cosmic ray physics may also directly contribute to a better understanding of the high-energy hadronic interaction in the forward region by specific investigations:

(i) Attenuation length and longitudinal development.

There are various EAS quantities which can be nearly directly related to ingredients of hadronic interaction models like the so-called hadronic interaction attenuation length $\Lambda$ to the inelastic cross section $\sigma_{p \text {-air }}^{\text {inel }}$, deduced e.g. from observations of the size spectra, registered with different angles of EAS incidence, i.e. with different atmospheric target thickness (see e.g. the approaches in Refs. [53,54]) and a recent discussion in Ref. [55]. In addition the longitudinal EAS development, which may be studied with optical detectors, is not only driven by the inelastic cross section of the primary particle, but also on cross sections of the secondary particles (pion-air cross sections) and the inelasticity $K$ of the hadron-air interactions. $K$ is the fraction of energy that is carried away by the produced secondary particles (see e.g. [17]).

(ii) Testing hadronic interaction models by EAS data.

Various (preferentially hadronic) observables, as predicted by the Monte-Carlo sim- 
ulations within the uncertainty of the primary elemental spectrum, can be compared with the measurements, thus shrinking the range of realistic models and pointing out obviously inconsistent model features $[33,56]$. The advantage of a multi detector experiment like KASCADE measuring simultaneously many EAS observables, is obvious. By applying a suitable discrimination method the $N_{e}-N_{\mu}$ correlation could be used to prepare event samples, which get highly enriched by EAS events induced by a defined (pure) mass group. For such enriched beams [57], other relevant observed EAS parameters could be studied and compared with simulations without the need of an a-priori assumption of a primary mass composition. The non-parametric procedures and applications (e.g. outlined in [20]) are of special interest.

(iii) Calorimetric measurements at high-mountain altitudes.

The feature that the most energetic particles in initial EAS stages are concentrated in the shower core is the basis of the traditional emulsion chamber experiments in high mountain altitudes on Mt. Chacaltaya or on the Pamir plateau [58].

A typical emulsion chamber set up consists at least of two lead-X-ray film sandwich chambers ( $\Gamma$ block and hadron block) of several $m^{2}$ area, separated by a layer of carbon and some spacer (see e.g. [59]). The radiation length in lead is very short $\left(6.37 \mathrm{~g} / \mathrm{cm}^{2}\right)$ compared to the nuclear interaction length $\left(\approx 150 \mathrm{~g} / \mathrm{cm}^{2}\right)$. Hence "atmospheric" photons and electrons initiate cascades very soon after entering the $\Gamma$ block on top. Hadrons on the other hand interact deeper in the chamber. Interactions of hadrons above the detector would be expected to produce both hadrons and photons. A pure electromagnetic cascade in the atmosphere would manifest itself in the chamber as a group of cascades ("families") all starting near the top of the chamber.

The potential of calorimetric devices installed in high mountain altitudes for studying hadronic interactions in energy ranges where collider experiments do not necessarily give the needed answers is rather promising. Nevertheless it is my feeling that the emulsion chamber technique has to be modernised towards the use of active detectors on high altitudes with a fast signal processing and event-visualisation. In addition such calorimeters should be embedded in a multi-detector array enabling adequate triggers and complete specifications of the observed events. Ideas in this direction and future prospects have been discussed in more detail for the case of Chacaltaya by Saavedra [60].

\section{Air fluorescence technique}

For the experimental EAS research at higher primary energies above $10^{17} \mathrm{eV}$ there is an efficient method applicable, which allows the measurement of the longitudinal development of the EAS, by air fluorescence observation. The technique relies on the fact that ionising particles can excite $N_{2}$ molecules in the atmosphere. Such excited molecules emit fluorescence photons (typically within $10 \mathrm{~ns}$ to $50 \mathrm{~ns}$ after excitation: Rigorously speaking it is luminescence). The optical fluorescence comes from various bands of molecular nitrogen of the molecular nitrogen ion, with light emitted between $3000 \AA$ and $4000 \AA$. It happens 


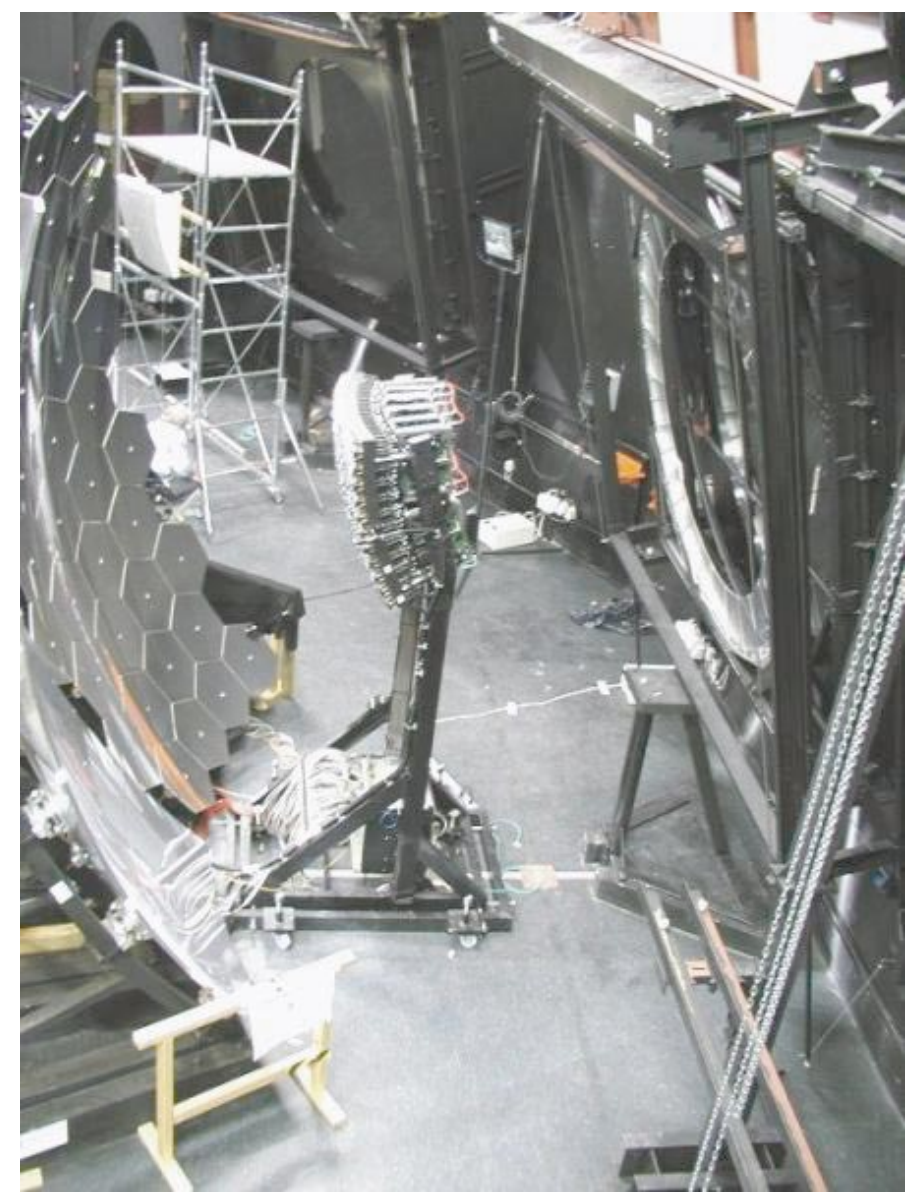

Figure 18: Fluorescence detector assembly [63].

to be just the wave band for which the atmosphere is quite transparent. The attenuation length is approximately $15 \mathrm{~km}$ for vertical incidence. The fluorescence yield per particle is weakly dependent on altitude and temperature. An EAS of $10^{17} \mathrm{eV}$ has more than 100 million electrons in the shower maximum, so that many fluorescence photons are generated, even with $0.5 \%$ fluorescence efficiency. The fluorescence light is isotropically emitted and can be detected at large distances from the shower axis. Thus it can be distinguished from air-Cherenkov light emitted in forward direction and confined to near distances from the shower axis. The detection problem is to identify the weak light traces, equivalent to a 40-Watt bulb, flying some microseconds through the atmosphere in several kilometers distance. The effective area for recording showers is very large as compared with conventional detector arrays and compensates the low duty cycle resulting from observations only during clear dark moonless nights. There are special techniques used for discrimination against night sky background and terrestrial sources of light noise (air planes, lightnings).

The fluorescence technique has been developed and effectively used by the Fly's Eye detectors, the original Fly's Eye [61] and the extension High Resolution Fly's Eye (HiResI+II) [62] installed in Dugway, Utah, $160 \mathrm{~km}$ from Salt Lake City, USA. 


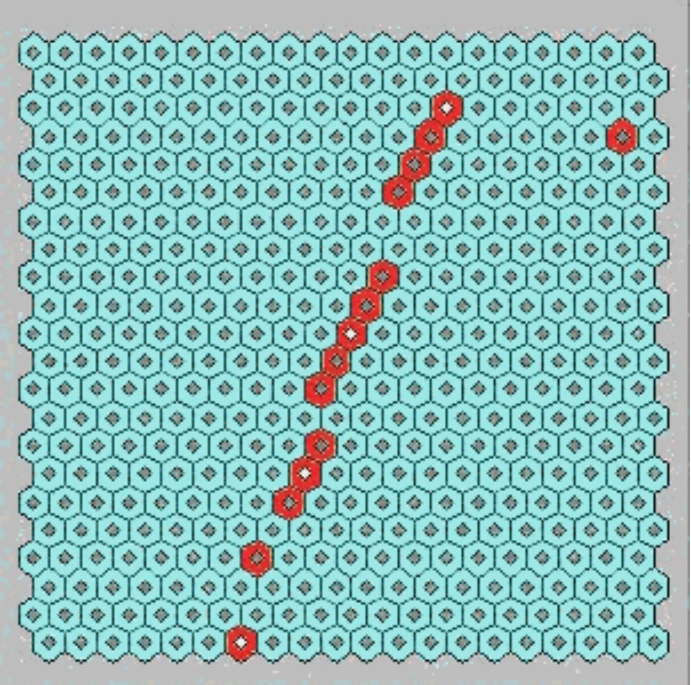

Figure 19: An EAS appears as a trace of illuminated pixels (photomultipliers) on the detector site. The line of the illuminated pixels determines the plane containing the detector and the EAS. In stereo observations by two fluorescence detectors at different sites the intersection of the two detector-EAS planes fixes the EAS geometry.

The fluorescence light - a flash of a few microseconds of duration - is collected using a lens or a mirror and projected onto a camera, located in the focal plane. Essentially the camera is an assembly of a large number of photo multipliers, each looking to a certain region of the sky. The camera pixelizes the image and records the time interval of the light arrival in each pixel element. Fig.18 displays an air fluorescence telescope [63] installed as a building stone of the Pierre Auger Observatory (PAO) [64] in Argentina for studying the highest energies.

The detector is a large area spherical mirror telescope of $11 \mathrm{~m}^{2}$ collecting area of aluminium segments, assembled with a correcting lens (Schmidt optics), covering a field of $30^{\circ} \cdot 30^{\circ}$. The camera is an assembly of 20 . 22 photomultipliers in the focus. The light trace is stored by a digital film in $100 \mathrm{~ns}$ distances.

Fig.19 shows the registered light trace of a cosmic particle, seen in $5.9 \mathrm{~km}$ distance in the Pampas recorded by the Auger prototype telescope at the inauguration of the Pierre Auger Observatory in June 2001. At some hexagonal pixels the trace was in between and the light could not activate the pixels. From such traces together with the timing information, or more accurately by a stereoscopic procedure using two telescopes, the longitudinal development (shower profile) of the extended air shower in the atmosphere could be reconstructed. The depth $X_{\max }$ of the shower maximum is sensitive to the primary mass $\left(X_{\max } \propto \ln \left(E_{0} / A\right)\right.$. The track length integral provides a calorimetric measure of the energy. It needs a calibration which involves necessarily a series of careful studies of the light production and transmission in the atmosphere (with monitoring), finally implying also some unknown dependence arising from the particle production model at highest energies.

\section{The ankle and above}

In the range of the highest energies the first remarkable feature, in fact finally establishing an enigma, comes from the existence of radiation fields in the cosmos, of which the $2.7 \mathrm{~K}$ microwave background is the best known, filling the entire universe with radio to far-infrared photons with a density of $\approx 400 \mathrm{~cm}^{-3}$. Above some energy thresholds the cosmic ray particles, coming from long distances, inelastically interact with those background photons. High-energy incident protons for which the background appear to be blue 
shifted, start photo-pion production (via exciting the $\Delta$ (1232)-resonance in nucleons, with less significance also by pair production $\left.p+\gamma_{2.7 K} \rightarrow p+e^{+}+e^{-}\right)$above a few tens of $E e V$ and get quickly cooled down in this way. This is the predicted Greisen-Zatsepin-Kuz'min $(\mathrm{GZK})$ spectral cut-off [3]. The consequence is that above $\approx 6 \cdot 10^{19} \mathrm{eV}$, photons, protons and nuclei have rather short attenuation lengths, in the order of, say several tens $M p c$ and the Universe gets relatively opaque for them. To state this more explicitly: It appears impossible for ultrahigh energy cosmic particles to reach us from sources whose distances would exceed $\approx 100 M p c[65]$ (this is roughly the size of our cosmic backyard i.e. the local supercluster of galaxies), unless rather exotic particles or exotic interaction mechanisms are envisaged. A second feature is related to the chemical composition of ultrahigh energy primary cosmic rays. If the highest energy cosmic rays would be mainly protons, as some experimental results are tentatively interpreted, the trajectories of single charged ultrahigh energy particles through the galactic and extra-galactic magnetic fields (which are believed to be in the order of $\mu G$ and $n G$, respectively) get no more noticeably deflected over distances limited by the Greisen-Zatsepin-Kuz'min (GZK) cut-off. Typically the angular deviation of a $10^{20} \mathrm{eV}$ proton from a source of $30 \mathrm{Mpc}$ distance would be about 2 degrees. In other words, above the cut-off, the direction of incidence of such particles (protons) should roughly point to the source, and to some extent proton astronomy should become possible, defined within the box of the consequences of the cut-off. However, looking in our astrophysical surroundings the number of adequate objects within a distance of a few $M p c$ and within the range of 2 degrees from arrival directions of registered ultrahigh-energy events is quite limited, if such objects would be even able to accelerate particles to such extremely high energies at all.

\begin{tabular}{llrl}
\hline \hline Tab.3. & & & UHECR detectors. \\
\hline \hline Array & Location & Area & Principal Detectors \\
\hline Volcano Ranch [63] & New Mexico, USA & $8 \mathrm{~km}^{2}$ & Scintillation \\
SUGAR [64] & Australia & $60 \mathrm{~km}^{2}$ & Muon \\
Haverah Park [14] & UK & $11 \mathrm{~km}^{2}$ & Water-Cherenkov \\
Yakutsk [15] & Russia & $10 \mathrm{~km}^{2}$ & Scintillation,Air-Cherenkov \\
AGASA [65,66] & Japan & $100 \mathrm{~km}^{2}$ & Scintillation,Muon \\
Fly's Eye [61] & Utah, USA & & Air fluorescence \\
HiRes(I+II)[62] & Utah, USA & & Air fluorescence \\
\hline \hline
\end{tabular}

What is the present experimental knowledge?

The data around the ankle and above stem from a few large-aperture ground based detector installations of two types of observation techniques: Surface detector arrays, which sample the secondary charged EAS particles on ground and air fluorescence detectors viewing light tracks in the atmosphere (see section 6). They are compiled in Tab.3.

UHECR research started with the smaller Volcano Ranch array nearly 40 years ago. 
There the first air shower event with the symbolic limit of $10^{20} \mathrm{eV}$ has been observed [66]. Lateron it was joined by the Sydney SUGAR array [67]. These detector arrays are mentioned in Tab.3 just from historical reasons.

The Haverah Park experiment in UK [14] was an array of water Cherenkov detectors, operated by the University of Leeds and other UK groups. The data collected from 1968 to 1987 are still basis of valuable analyses of EAS above energies of $6 \cdot 10^{16} \mathrm{eV}$. In context with the Haverah Park experiment the method of using the charge particle density, registered at the distance of $600 \mathrm{~m}$ from the EAS axis, has been developed for the determination of the primary energy [48]. Due to reduced shower fluctuations of the density of particles (preferentially muons) at larger distances and, as simulations show, due the insensitivity to the primary mass and the specific interaction model, this parameter $S(600)$ has been proven to be very robust energy estimator.

The technique has been essentially adopted by the Yakutsk array [15] and the AGASA experiment [68,69]. The Yakutsk array in Russia is additionally equipped with 50 Cherenkov detectors for studying the Cherenkov light emission and checking the $S(600)$ method for the determination of the primary EAS energy. The AGASA experiment located in the Akeno observatory in Japan covers an area of $100 \mathrm{~km}^{2}$ and is presently the world's largest detector array in operation. For the energy calibration via $S(600)$ the AGASA studies take into account the attenuation of $S(600)$ for inclined showers which pass through a larger atmospheric thickness [69]. In context with a global analysis of data from the Yakutsk array the question of the energy determination by $S(600)$ has been recently scrutinised on basis of QGSJET Monte-Carlo simulations [70].

The Fly's Eye [61] in Dugway, Utah, USA, was the first successful air fluorescence detector and demonstrated the power of the technique of primary energy and mass determination. HiRes [71] collects for each pixel of the fluorescence detectors the pulse height and arrival time, from which the EAS geometry can be reconstructed (see section 6). It consists of two detector installations located in a distance of $12.6 \mathrm{~km}$ separated from each other. An important feature of the High Resolution Fly's Eyes detectors (HiRes-I and HiRes-II) [62] is that showers viewed by both detectors ("stereo events") can be measured with significant redundancy.

\subsection{Structure of the spectrum: Cut-off or not?}

Fig.20 displays the highest energy region of the cosmic ray spectrum as observed by the AGASA detector $[4,72,73]$. The digits near the data points indicate the number of events and the error bars show the $90 \%$ confidence level. The energy spectrum is multiplied by $E^{3}$, so that the part below $10^{18} \mathrm{eV}$ becomes flat. There is also a dip centered around $3 \cdot 10^{18} \mathrm{eV}$ which is a significant feature and due to a change in the spectral slope. The ankle structure becomes evident with a deviation from the cut-off predictions i.e. an excess of ultrahigh-energy events as compared with the spectrum expected from distant sources. The theoretical curve which anticipates the GZK cut-off mechanism and an adopted uniform distribution of the sources, indicates that the expected GZK cut-off is not an abrupt drop down, rather a suppression of the ultrahigh-energy flux. Near the GZK cut-off energy there should be also an enhancement expected due to a "pileup effect" of cosmic particles 


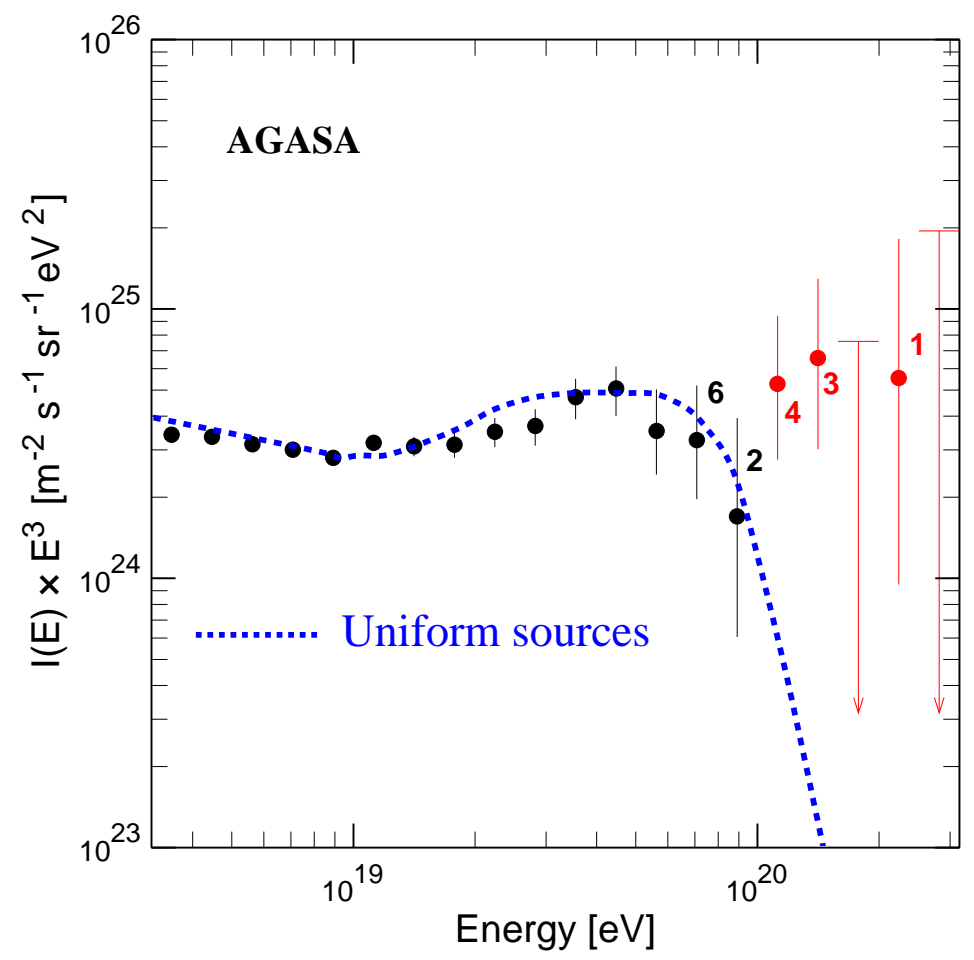

Figure 20: Highest energy region of the cosmic ray spectrum [72,73].

starting with higher energies and crowding up in energy just below the cut-off. There are of course large error bars, but the tendency of the existence of a trans-GZK cosmic flux could be considered as established when other events are included from other detectors (see the review of Nagano and Watson [74]). In the past also Fly's Eye and the HiRes collaboration have reported a number of trans-GZK cut-off events (see e.g. [75,76]).

Thus, though the experimental basis was still poor and under debate, till 2001 there seemed to be a, may be somehow biased, but tacit common understanding on the fact that the ultrahigh-energy cosmic ray spectrum extends to energies $>10^{20} \mathrm{eV}$. However, after a re-calibration by the HiRes collaboration with withdrawing some results, a discussion during the last International Cosmic Ray Conference in Hamburg 2001 made obvious that there is a disagreement of different observations, between the AGASA and HiRes results, in particular, concerning the inferred fluxes and the energy calibration. Though this disagreement has been subsequently somehow relieved by a serious revision of the previously reported events, it is not definitively removed. In recent papers of the HiRes collaboration $[77,78]$ the current situation is discussed.

Fig.21 compiles the recent status comparing the monocular spectra from both the HiRes-I and HiRes-II detectors [77,78] with the latest results of the AGASA experiment. The highest energy event observed with HiRes is at the reconstructed energy of $1.84 \cdot 10^{20} \mathrm{eV}$. The AGASA results, most recently updated [79] have excluded events with zenith angles larger than $45^{\circ}$, and they differ from the communication at the 27th ICRC in Hamburg 


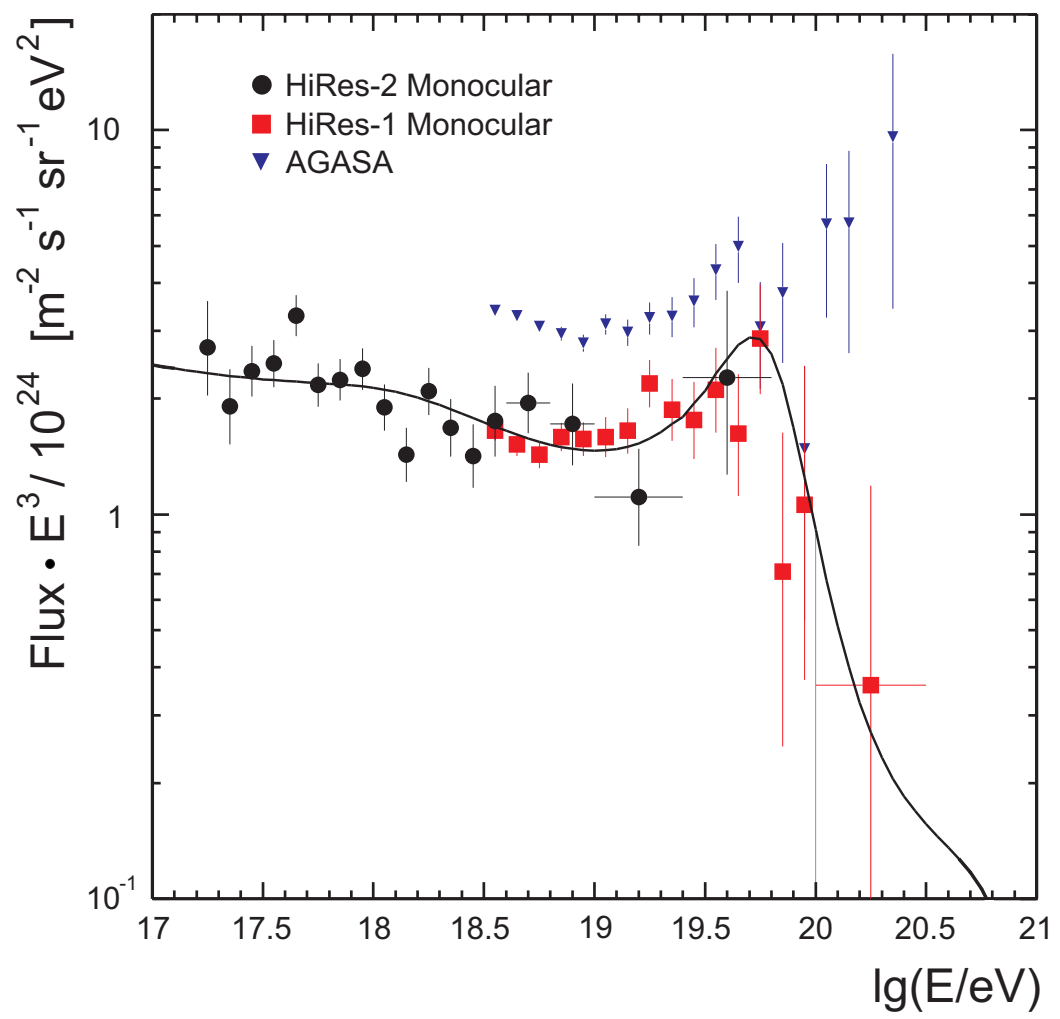

Figure 21: Ultra High Energy Cosmic Ray Flux: Comparison of the results of HiRes and AGASA. The HiRes results which appear to be consistent with the GZK cut-off have been analysed in terms of a model with uniformly distributed proton sources [78].

2001 [69]. Obviously below $10^{20} \mathrm{eV}$ the AGASA flux consistently differs from the HiRes observations, which appears to be pronounced in the $I(E) \cdot E^{3}$ presentation in the figure. In fact a $20 \%$ lowering of the energy scale of the AGASA experiment would reduce this disagreement considerably. The HiRes spectrum appears consistent with existence of the GZK cut-off. The line represents a fit assuming the GZK cut-off (with energy losses by pion- and $e^{+} e^{-}$-production with the cosmic microwave background radiation) and a model of galactic and extragalactic sources, uniformly distributed across the Universe [80]. Above $10^{20} \mathrm{eV}$ the HiRes spectrum, as it stands, and also the re-analysed spectrum communicated by the Yakutsk Experiment [81], significantly differ from that reported by the AGASA experiment. But it should be also noted that a critical parameter for the calibration of the fluorescence observations is the fluorescence yield, which turned out to be not known with sufficient accuracy. A critical discussion of this present observational situation with the question "Has the GZK cut-off been discovered?" is given by Bahcall and Waxman [6] concluding that the existing data leave the question still open.

\subsection{Elemental composition}

Fig.22 compiles recent experimental information about the variation of the mass composition of the ultrahigh-energy cosmic rays. This information is carried by the position of 


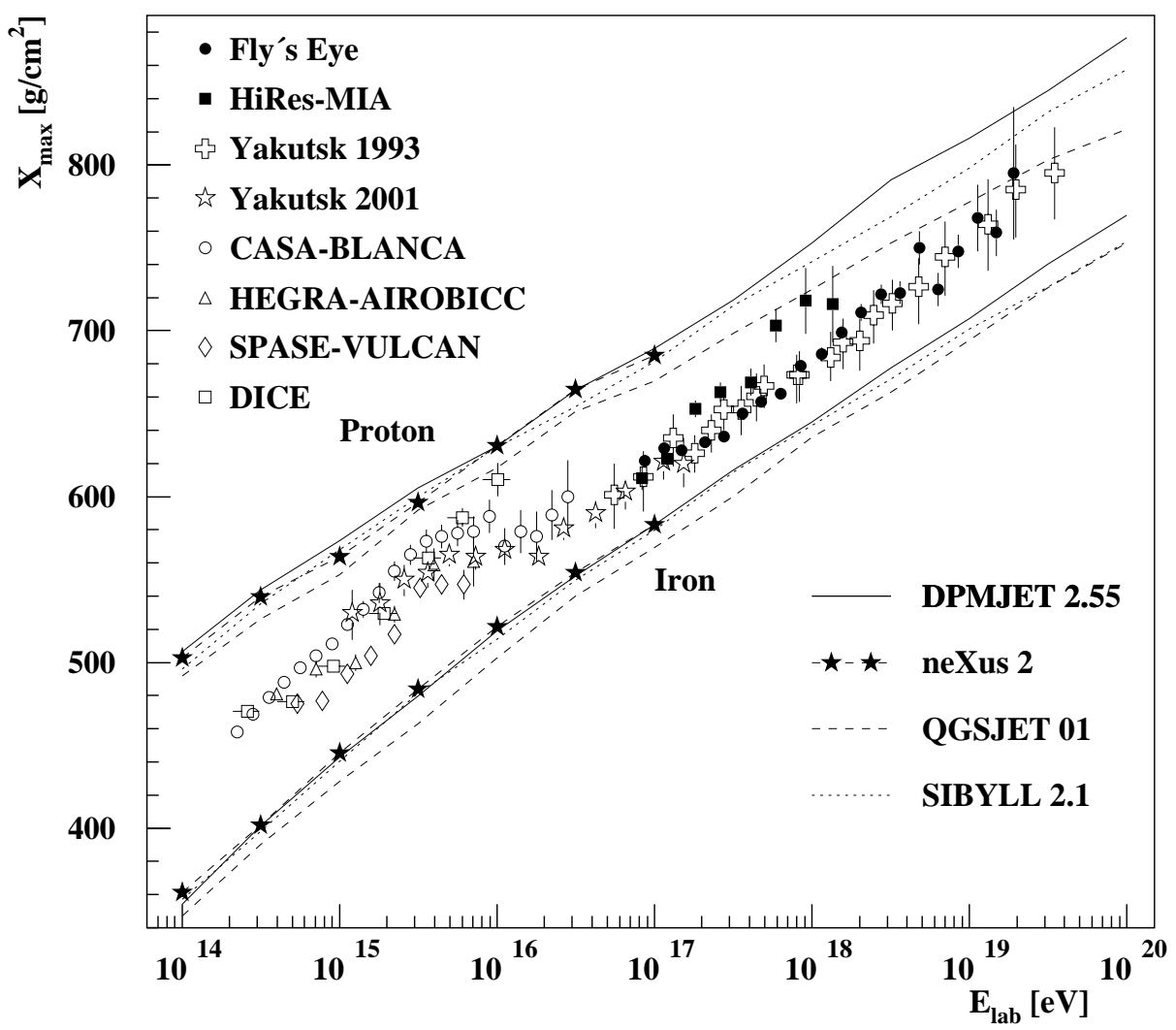

Figure 22: Average depth $X_{\max }$ of the EAS maximum vs. the primary energy from various measurements [18,82]. The experimental results are compared to theoretical predictions (of four different hadronic interaction models) for proton (upper curves) and iron (lower curves) primaries.

the shower maximum $X_{\max }$, which can be determined either from the reconstruction of the longitudinal EAS development by the air fluorescence technique or from the lateral distribution of the Cherenkov light.

The mass discrimination effect arises from fact that protons probe deeper in the atmosphere and their EAS develop more slowly than expected for heavy nuclei of the same energy. Thus a change in the elongation rate (that is logarithmic change of $X_{\max }$ with the energy) indicates a change in the mass composition. However this feature needs also a calibration by referring to a hadronic interaction model for the Monte-Carlo simulations. The theoretical curves in Fig.22 display the model dependence. They include the predictions by a new event generator neXus2 with great universality [83]. Qualitatively the mass composition appears to be mixed or heavy just above the knee with a global trend, starting around a few $10^{17} \mathrm{eV}$, towards a light composition in the energy range around $30 \mathrm{EeV}$.

\subsection{Arrival directions}

In 1999 Hayashida et al. [84] reported a small directional anisotropy for EAS events in the energy range of $1 \cdot 10^{18} \mathrm{eV}$ to $3 \cdot 10^{18} \mathrm{eV}$, which may provide evidence that $\mathrm{EeV}$ cosmic rays have sources in our galaxy. This finding is corroborated by observations of the Fly's Eye 
[85]. In contrast above $10^{19} \mathrm{eV}$ cosmic rays display apparent isotropy [73] i.e. a distribution of the arrival directions without any significant galactic-plane enhancement, to the extent to which such conclusions can be drawn with statistical significance in the moment. This feature together with the trend to a light mass composition provides reasonable arguments that an extragalactic cosmic ray component comes into play at the highest energies.

But present observational situation is characterised by many experimental uncertainties, and the current discussion is dominated by the quest for a higher statistical and systematic accuracy to prove or to disprove the actual more or less bold conjectures. This implies consequently a call for new powerful experimental installations.

\section{Physical and astrophysical implications of a trans-GZK cut-off flux}

The existence of UHECR events constitutes an enigma by the question: Where are the sites and what are the acceleration mechanisms being capable to impart energies of macroscopic orders to a microscopic particle? Many processes have been proposed (see [86]) where in an astrophysical plasma large-scale macroscopic motion is transferred to individual particles, for example in a turbulence and by shock waves. The crucial role plays the size $L$ of the acceleration region and the magnetic field $B$ embedded in the plasma and keeping the gyroradius of the particle in the acceleration region. That depends also on the velocity $\beta$ of the motion (in the conventional approach of shock wave acceleration by the Fermi mechanism, say in supernova remnants, $\beta$ is in the order of 0.01 ). Traditionally astrophysical accelerator candidate sites are compiled by a $B$-vs.- $L$-plot introduced by M. Hillas [87] and often adopted in various modifications. It is constructed according to an estimate for the maximum total energy $E_{\max } \propto \beta \cdot Z e \cdot B \cdot L$ of the particles, neglecting energy losses from synchrotron radiation or the interaction with the microwave background. This dimensional constraint rules already out most astronomical objects. Possible objects include radio galaxies, neutron stars and Active Galactic Nuclei. Additionally gamma-ray bursts as sources of zevatrons $\left(1 \mathrm{ZeV}=10^{21} \mathrm{eV}\right)$ have been discussed ([88], see also [89]). If all parameters related to the question are taken into account, one has to concede that none of the proposed scenarios is fully convincing. In addition we have to keep in mind that the sources should be nearby in cosmological scales. Within the present statistical accuracy the data do also not show a distinct correlation with nearby point sources. There are various examinations of the arrival directions of the highest energy events (see [86]). Ahn et al. [90] proposed a galactic wind model for the local magnetic fields and traced back trans-GZK cut-off events within $20^{\circ}$ of the active galaxy $M 87$ in the Virgo cluster (about $20 \mathrm{Mpc}$ away). They had to assume that the two cosmic particles registered with the highest energies $\left(2 \cdot 10^{20} \mathrm{eV}\right.$ and $\left.3.2 \cdot 10^{20} \mathrm{eV}\right)$ are He particles. In fact, there is a suggestion for the origin of trans-GZK cut-off events that they are produced by heavy nuclei, since the energy loss times for heavy ions of energies $>3 \cdot 10^{20} \mathrm{eV}$ are longer than for protons [91]. In case that the hypothesis of a galactic wind accelerator would be turn out to be real, proven by larger statistical accuracy of the observations, a near-by gigantic accelerator would have been discovered, reaching energies many orders of magnitude higher than any conceivable man-made machine. 
However, if future studies would exclude "conventional" astrophysical acceleration mechanisms, one would need to consider another class of theories proposed as possible explanation, so-called top-down processes. Most of those speculations study the possibility that UHECR arise from the decay of some super-heavy X particles whose mass is in the Grand Unification range $\left(10^{25} \mathrm{eV}\right)$ produced during a phase transition period or by topological defects during the early Universe. The models differ mainly in how to produce the density of X particles to fit the UHECR observations and their survival from some $10^{-35} \mathrm{~s}$ after Big Bang. An extensive review about top-down models is published by Bhattacharjee and Sigl [92] with an exhaustive list of references. Great interest has been induced by the idea of Z-bursts that extremely ultrahigh-energy neutrinos $(\approx 10 \mathrm{ZeV})$ could produce ultrahigh-energy Z by interactions with thermal background neutrinos (see Z. Fodor, these proceedings). However, with such a conjecture the problem appears shifted to the question of a source of $10 \mathrm{ZeV}$ neutrinos. It has been also suggested that at ultrahigh energies the neutrino nucleon cross section would increase to hadronic values $(100 \mathrm{mb})$, so that neutrinos could produce giant air showers ([93], see S. Kovesi-Domokos, these proceedings). Another unconventional idea, which has been already discussed in the 70ties [94], is that the Lorentz invariance might be weakly broken at ultrahigh energies so that the photo-pion production and the GZK cut-off get suppressed.

One should mention that such speculative models and the numerous ideas en vogue, arising with the prospect of new physics and new astrophysics, have quite specific features and experimental signatures (shape of the spectrum and mass composition) so that a discrimination appears to be not impossible, provided the experimental knowledge could get sufficiently increased. For example, the presence or absence of the pileup at the predicted GZK cut-off energy could be a signature for bottom-up or top-down models, respectively. Top-down scenarios are expected to be signalled by an increased amount of high-energy photons.

\section{The next and over-next generation of detectors}

The AGASA ground based array and the HiRes fluorescence detectors are continuing to collect data on ultrahigh energy EAS. The Japanese Telescope Array is planned and operated as array of several telescopes for gamma-ray and UHE neutrino, but also for UHECR observations [95].

The community looks forward with great interest to the next detector: The Pierre Auger Observatory [64] with $14000 \mathrm{~km}^{2} \mathrm{sr}$ aperture over two sites, one in each hemisphere. The installation of the southern Pierre Auger Observatory (Fig.23) has started in 2000 with a prototype array of $55 \mathrm{~km}^{2}$ and an air fluorescence telescope, near the small town of Malargüe in the province of Mendoza, Argentina. In final the site will be equipped with 1600 detector stations $\left(12 \mathrm{~m}^{3}\right.$ tanks filled with water detecting the Cherenkov light produced in the water tanks by secondary particles), distributed in a grid with $1.5 \mathrm{~km}$ spacing. The stations of the surface array will be operated by battery backed solar power and will communicate with the central station by wireless links. Four eyes (installed at the periphery), composed altogether of 24 air fluorescence telescopes, will view $8000 \mathrm{~km}^{2}$ of 


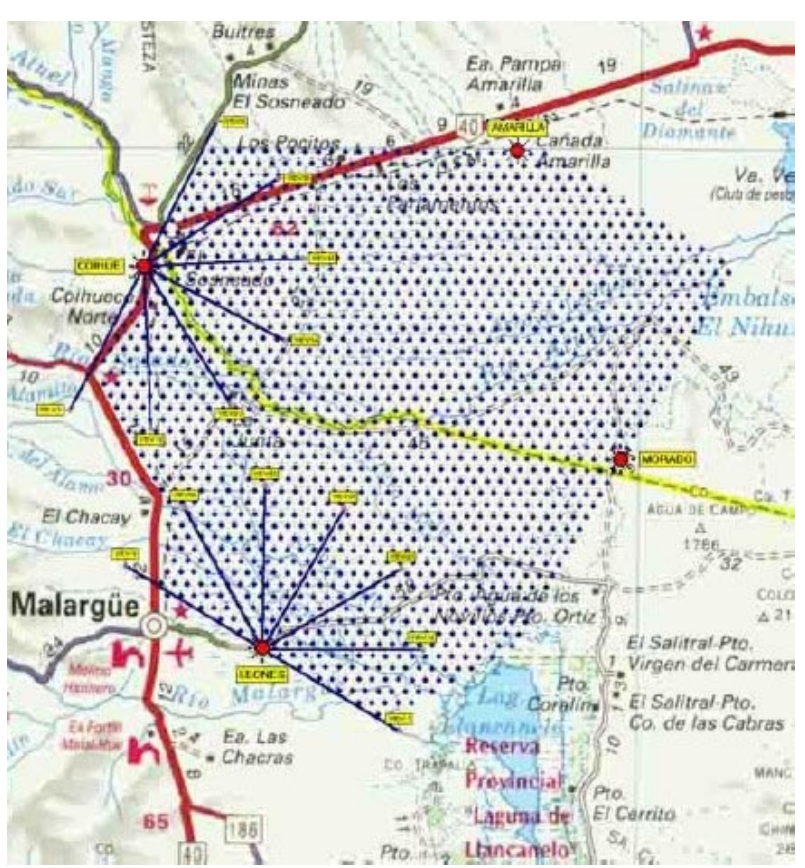

Figure 23: Layout of the southern Pierre Auger Observatory.

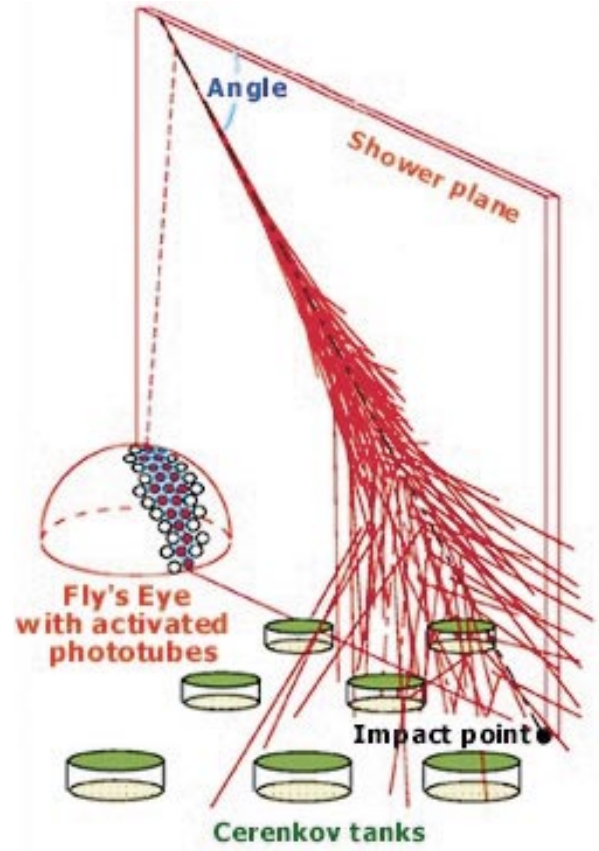

Figure 24: Hybrid detector concept.

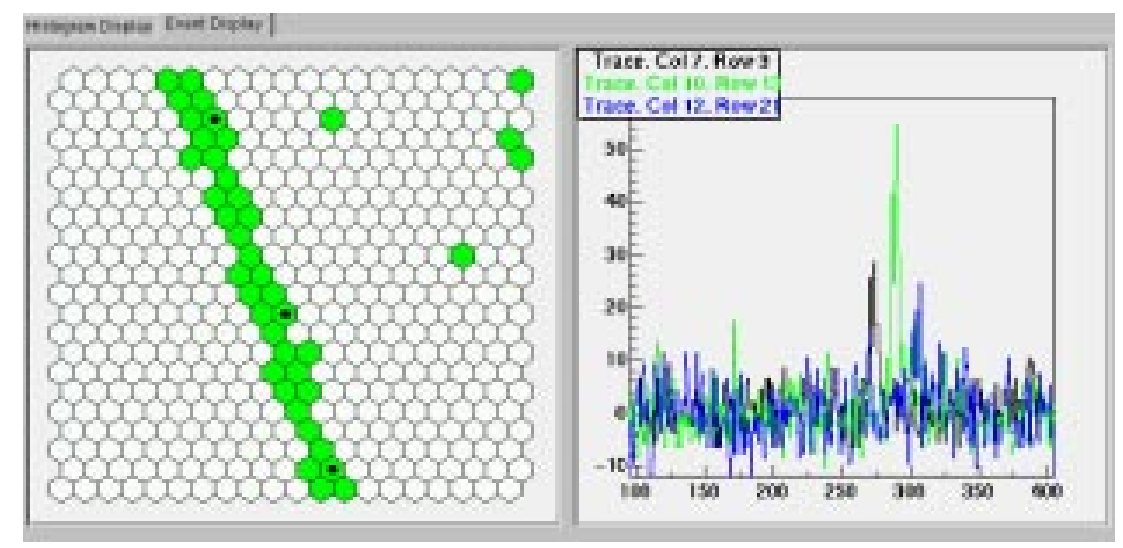

Figure 25: Display of an event registered with the prototype air fluorescence telescope. In addition to the trace in the camera the time variation of the amplitude is shown [96]. The time scale is in ns.

the site and measure during clear moonless nights i.e. with a duty cycle of $10 \%$ the giant showers through the air fluorescence.

The concept (Fig.24) of an hybrid detector provides unique advantages. A subsample of $10 \%$ of the total number of events simultaneously observed with both techniques, enables a cross calibration and yield an unprecedented quality for shower identification. It is expected to detect some 60 to 100 events per year above $10^{20} \mathrm{eV}$, and 100 times more above $10^{19} \mathrm{eV}$. Fig. 25 displays a hybrid event as registered by the prototype fluorescence telescope in February 2002 [96]. 


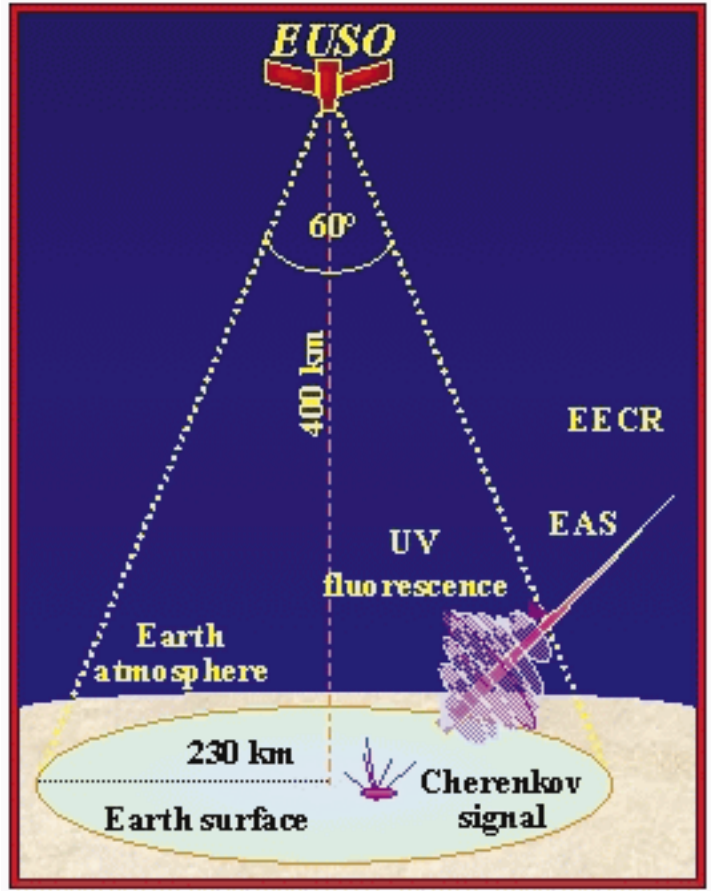

Figure 26: Principle of the EUSO detector [97].

The Pierre Auger Observatory has just started with prototype observations, and the community looks already forward to the next generation of detectors. There is little doubt that the next big step will be an air-borne detector observing the giant shower development in the atmosphere with a huge aperture down to the Earth's atmosphere.

This is envisaged with the OWL (Orbiting Wide angle Light Collectors) Airwatch mission by fluorescence detectors carried on two satellites in a low Earth orbit $(600 \mathrm{~km}-1200 \mathrm{~km})$ and observing (in a stereoscopic mode) giant EAS from space [97], also with the perspective to open the way for neutrino astronomy in the high-energy range. Operation from space dramatically increases the active volume and the observed EAS event rate. The OWL is expected to be able to detect more than 1000 EAS per year of energies above $10^{20} \mathrm{eV}$, provided the spectrum follows the trend of the AGASA results.

At present there is feasibility study of the European Space Agency considering an Extreme Universe Space Observatory (EUSO) to install such a detector (Fig.26) on the International Space Station and to develop the technology. Another proposal of this kind is a balloon borne project using the Antarctic ice as reflector of Cherenkov light [98].

\section{Concluding remarks}

The future projects are of particular interest when in few years the spectrum would have been shown to be exceeding the reach of the Pierre Auger Observatory, and again larger statistical accuracies would be necessary for studies following the focal points of Extremely High Energy Cosmic Ray Observations (Tab.4).

A lesson of the advanced studies of the knee region, as done, for example, with KASCADE is that investigations of far-reaching physical and astrophysical aspects by EAS observations have to be accompanied by a serious and quantitative understanding of the particle interactions in that energy range. That challenging task, which cannot be disentangled from the astrophysical aspects, is the other side of the medal of necessary efforts with interesting aspects. Without knowing the interactions of the unknown particles, even the energy estimation of giant EAS and the scale of the spectrum will remain finally under debate! It is fair to note that many analyses of UHECR EAS events and confusing results suffer from the use of less mature interaction models and that an impact like that of COR- 
SIKA for the research at the knee is needed.

\begin{tabular}{l}
\hline \hline Tab.4. \\
\hline \hline - The change of the spectral index at the ankle. Change in the production \\
mechanism? Change in the elemental composition? Change in the interaction \\
processes? \\
- Shape of the energy spectrum of the highest energies. What is the \\
maximum cosmic ray energy? Is there any limit? Evidence for a pileup bump \\
at the end? Evidence of the existence or non-existence of the GZK cut-off? \\
What is the mass of the primary particles of highest energies? \\
- Search for signatures of the origin of UHECR. Bottom-up acceleration \\
or new physics of top-down processes, only few decades below the Grand \\
Unification energy?
\end{tabular}

With such caveats in mind the prevailing understanding of the present situation may be characterised as follows: The most remarkable feature of the cosmic radiation is that the investigations have not yet found a definite natural end of the energy spectrum. Anticipating the spectrum would extend beyond the GZK cut-off, there is a plethora of interesting speculations, but presently we are not able to identify or clearly specify signatures of the origin and of cosmic sources of such a trans-GZK radiation. The features of the present observations do establish an actual mystery of great cosmological relevance at the frontier of natural science.

\section{Acknowledgments}

I would like to thank Otto Nachtmann and Karlheinz Meier for the opportunity to present this overview at the 26th Johns Hopkins Workshop on Current Problems in Particle Theory, Heidelberg, August, 2002. I am particular grateful to Ralph Engel and Andreas Haungs for many clarifying discussions about the current status of the subject, and Mashimuro Teshima for private communications. Finally I would like to acknowledge the expert help of Jürgen Oehlschläger in preparing the script.

\section{References}

[1] G.V. Kulikov and G.B. Khristiansen, Sov. Phys. JETP 35 (1959) 441

[2] H.O. Klages et al. - KASCADE Collaboration, Nucl. Phys. B Proc. Suppl. 52B (1997) 92

[3] K. Greisen, Phys. Rev. Lett. 16 (1966) 748; G.T. Zatsepin and V.A. Kuz'min, Zh. Eksp. Teor. Fiz., Pisma Red.4 (1966) 144 (Sov. Phys. JETP Lett. 4 (1966) 78)

[4] M. Takeda et al. - AGASA Collaboration, Phys. Rev. Lett. 81 (1998) 1163 
[5] D.J. Bird et al., Ap.J. 441 (1995) 144 Ch.C.H. Jui - HiRes Collaboration, Highlight paper at the 26th ICRC, Salt Lake City, USA, 1999, AIP Conference Proceedings, eds. B.L. Dingus, D.B. Kieda, M.H. Salamon vol. 516, p.370

[6] J.N. Bahcall and E. Waxman, hep-ph/0206217 (2002)

[7] P. Auger, R. Maze and T. Grivet-Mayer., C.R. Acad.Sci.Ser. 2206 (1938) 172; ibid 207 (1938) 228

[8] W. Kohlhörster, I. Matthes und E. Webber, Naturwissenschaften 26 (1938) 576

[9] H. Engler et al., Nucl. Instr. Meth. A427 (1999) 528

[10] H. Bozdog et al., Nucl. Instr. Meth. A465 (2001) 455

[11] P. Doll et al., Nucl. Instr. Meth., A488 (2002) 517

[12] H. Rebel, Nucl. Phys. A 663+664 (2000) 847c

[13] M. Nagano et al., J. Phys. G: Nucl. Phys. 10 (1984) 1295

[14] M.A. Lawrence, R.J.O. Reid, A.A. Watson, J. Phys. G: Nucl. Part. Phys. 17 (1991) 773; Proc. 21st ICRC, 3 (1990) 159

[15] N.N. Afanasiev et al., Proc. 24th ICRC, Rome, Italy, 2 (1995) 756; N.N. Efimov et al., Proc. Internat. Symposium on Astrophysical Aspects of the Most Energetic Cosmic Rays, 1991, eds. M. Nagano and F. Takahara (Singapore: Word Scientific), p.20

[16] D. Heck et al., FZKA Report 6019, Forschungszentrum Karlsruhe 1998

[17] T. Stanev, Rapporteur talk at the 26th ICRC, Salt Lake City, USA, 1999, AIP Conference Proceedings, eds. B.L. Dingus, D.B. Kieda, M.H. Salamon, vol. 516, p.247

[18] R. Engel, Invited, Rapporteur, and Highlight Papers, 27th ICRC, Hamburg, Germany, 2001, ed. R. Schlickeiser, p. 181

[19] A.A. Chilingarian, Compt. Phys. 54 (1989) 381; A.A. Chilingarian et al., Nucl. Phys. B Proc. Suppl. 52B (1997) 237

[20] T. Antoni et al. - KASCADE Collaboration, Astrop. Phys. 16 (2002) 245

[21] M. Roth et al. - KASCADE Collaboration, Proc. 27th ICRC, Hamburg, Germany, 1, (2001) 88

[22] L.O.C. Drury, Rep. Progr. Phys. 46 (1983) 973; in Nuclei Far from Stability and Astrophysics, NATO Science Series II Vol. 17, eds. D.N. Poenaru, H. Rebel, J. Wentz, Kluwer Academic Publishers, Dordrecht, p.341

[23] J.G. Kirk and R.O. Dendy, J. Phys. G: Nucl. Part. Phys. 27 (2001) 1589

[24] C.J. Cesarsky, Ann. Rev. Astron. Astrophys. 18 (1980) 289

[25] A.D. Erlykin and A.W. Wolfendale, J. Phys. G: Nucl. Part. Phys. 23 (1997) 979

[26] S.I. Nikolsky, Nucl. Phys. Proc. Suppl. A39 (1995) 157

[27] J. Hörandel et al. - KASCADE Collaboration, Proc. 27th ICRC, Hamburg, Germany, 1, (2001) 137

[28] A. Haungs et al., Nucl. Instr. Meth. A372 (1996) 515 
[29] H. Rebel, G. Völker, M. Föller and A.A. Chilingarian, J. Phys.G: Nucl. Part. Phys. 21 (1995) 451

[30] C. Büttner et al. - KASCADE Collaboration, Proc. 27th ICRC, Hamburg, Germany, 1 (2001) 153

[31] N.N. Kalmykov, S. Ostapchenko and A.I. Pavlov, Nucl. Phys. B Proc. Suppl. 52B (1997) 17

[32] K. Werner, Phys. Rep. 232 (1993) 87

[33] T. Antoni et al. - KASCADE Collaboration, J. Phys. G: Nucl. Part. Phys.25 (1999) 2161

[34] M. Nagano et al., J. Phys. G: Nucl. Part. Phys. 18 (1992) 423

[35] M. Amenomori et al., Ap. J.461 (1996 ) 408

[36] M.A.K. Glasmacher et al., Astropart. Phys. 10 (1999) 291

[37] M. Roth et al. - KASCADE Collaboration, Proc. 12th ISVHECRI, CERN 2002, to be published in Nucl. Phys. B Proc. Suppl.

[38] K.-H. Kampert et al. - KASCADE Collaboration, Invited Rapporteur, and Hightlight papers at the 27th ICRC, Hamburg, Germany 2001, ed. R. Schlickeiser, p. 240

[39] M. Bertaina et al. - KASCADE-Grande Collaboration, Proc. 27th ICRC, Hamburg, Germany, 2 (2001) 792

[40] J.R. Patterson and A.M. Hillas, J. Phys. G: Nucl. Phys. 9 (1983) 1433

[41] A. Lindner et al., Astrop. Phys. 8 (1998) 235

[42] J.W. Fowler et al., Astrop. Phys. 15 (2001) 49

[43] S. Paling et al., Proc. 25th ICRC, Durban, RSA, 5 (1997) 253

[44] K. Boothby et al., Ap. J. 491(1997) L35

[45] F. Aqueros et al., Astronomy and Astrophysics 359 (2000) 682

[46] H.E. Dickinson et al., Proc. 26th ICRC, Salt Lake City, USA, 3 (1999) 136

[47] M.N. Dyakonov et al., Proc. 23th ICRC, Calgary, Canada, 4 (1993) 303

[48] A.M. Hillas, D.J. Marsden, J.D. Hollows and H.W. Hunter, Proc. 12th ICRC, Hobart, Australia, 3 (1971) 1001; A.M. Hillas, Proc. 17th ICRC, Paris, France, 8 (1981) 1993

[49] S.J. Sciutto, astro-ph/9911331

[50] R.S. Fetcher et al., Phys. Rev. D 50 (1994) 5710

[51] J. Ranft, hep-ph/9911213 (1999)

[52] L. Jones, CERN COURIER 42, No. 6 (July/August 2002) 26; http://www-ik.fzk.de/ needs/

[53] G. Maier et al. - KASCADE Collaboration, Proc. 27th ICRC, Hamburg, Germany, 1 (2001) 161

[54] A.A. Chilingarian et al., Proc. 27th ICRC, Hamburg, Germany, 1 (2001) 165

[55] J. Alvarez-Muñiz et al., Phys. Rev. D 66 (2002) 123004

[56] T. Antoni et al. - KASCADE Collaboration, J.Phys G: Nucl. Part. Phys. 27 (2001) 1785 
[57] A.A. Vardanyan et al. - KASCADE Collaboration, Proc. 27th ICRC, Hamburg, Germany, 1 (2001) 67; T. Antoni et al. - KASCADE Collaboration, Enriched cosmic ray mass groups with KASCADE, in preparation

[58] L.T. Baradzei et al. - Chacaltaya and Pamir Collaboration, Nucl. Phys. B370 (1992) 365

[59] S.L.C. Barroso et al., Proc. 26th ICRC, Salt Lake City, Utah, USA, 1 (1999) 119

[60] O. Saavedra, in "Nuclei Far from Stability and Astrophysics", NATO Science Series II vol. 17, eds. D.N. Poenaru, H.Rebel, J.Wentz, Kluwer Academic Publishers, Dordrecht, p.385

[61] R.M. Baltrusaitis et al., Nucl. Instr. Meth. A240 (1985) 410

[62] J.N. Matthews et al. - High Resolution Fly's Eye Collaboration, Proc. 27th ICRC, Hamburg, Germany, 2 (2001), 350

[63] Forschungszentrum Karlsruhe: Press Information 2001/PI212001; http://www.fzk.de

[64] Pierre Auger Project Design Report 1997, Pierre Auger Collaboration, Fermi National Accelerator Lab.; M.T. Dova et al. - Pierre Auger Observatory Collaboration, Proc. 27th ICRC, Hamburg, Germany, 2 (2001) 699; http://www.auger.org/admin/DesignReport/index.html

[65] T.Stanev et al., Phys Rev. D 62 (2000) 093005

[66] J. Linsley, Phys. Rev. Lett. 10 (1963) 146; Proc. 8th ICRC, Jaipur, India, 4 (1963) 295

[67] M.M. Winn et al., J. Phys. G: Nucl. Phys. 12 (1986) 653

[68] S. Yoshida et al., Astropart. Phys. 3 (1995) 105

[69] N. Sakaki et al., Proc. 27th ICRC, Hamburg, Germany, 1 (2001) 333

[70] A.V. Glushov et al., Physics of Atomic Nuclei, 65 (2002) 1313

[71] G.L. Cassidy, Rev. Nucl. Part. Sci. 35 (1985) 351

[72] M. Takeda et al., Astrophys. J. 522 (1999) 225

[73] M. Takeda et al., astro-ph/0209422

[74] M. Nagano and A.A. Watson, Rev. Mod. Phys. 72 (2000) 689

[75] D.J. Bird et al., Phys. Rev. Lett. 71 (1993) 3401

[76] Ch.C.H. Jui - HiRes Collaboration, Highlight paper at the 26th ICRC, Salt Lake City 1999, AIP Conference Proceedings, eds. B.L. Dingus, D.B. Kieda, M.H. Salamon, vol. 516, p.370

[77] T. Abu-Zayyad et al., Phys. Rev. Lett. - astro-ph/020843

[78] T. Abu-Zayyad et al., Astropart. Phys. - astro-ph/0208301

[79] M. Teshima, private communication; M. Takeda et al., Astropart. Phys. - astro-ph/0209422

[80] V. Berezinski, A.Z. Gazizov, and S.I. Grigorieva, hep-ph/0204357

[81] M.I. Pravdin et al., Proc.26th ICRC, Salt Lake City, USA, 3 (1999) 292; E.E. Antonov et al., Sov. Phys. JETP Lett. 69 (1999) 650

[82] D. Heck et al., Proc. 27th ICRC, Hamburg, Germany, 1 (2001) 233;

[83] H.J. Drescher et al., Phys. Rep. 350 (2001) 93

[84] N. Hayashida et al., Astropart. Phys. 10 (1999) 303 
[85] H. Dai et al. Astrophys. J. 511 (1999) 739

[86] P.L. Biermann, E.-J. Ahn, G. Medina-Tanco, T. Stanev, 7th course, Current Topics in Astrofundamental Physics, Erice, Dec. 1999; http://www.mpifr-bonn.mpg.de/div/theory/

[87] A.M. Hillas, Ann. Rev. of Astronomy and Astrophysics, 22 (1984) 425

[88] E.W. Waxman, Phys. Rev. Lett. 75 (1995) 386

[89] F.W. Stecker, astro-ph/0207629 /2000

[90] E.-J. Ahn, G. Medina-Tanco, P.L. Biermann, T. Stanev, Phys. Rev. Lett., astro-ph/9911123

[91] F.W. Strecker and M.H. Salamon, Astrophys.J. 512 (1999) 521

[92] P. Bhattarcharjee and G. Sigl, Phys. Rep. 327 (2000) 109

[93] G. Domokos and S. Kovesi-Domokos, Phys. Rev. D 38 (1988) 2933

[94] H. Sato and T. Tati, Prog. Theor. Phys. 47 (1972) 1788

[95] M. Teshima et al., Nucl. Phys. B Proc. Suppl. 28B (1992) 169; T. Yamamoto et al. - Seven Telescope Array Collaboration, 26th ICRC, Salt Lake City, USA, 5 (1999) 275

[96] H.J. Mathes, private communication

[97] L. Scarsi et al. - OA Collaboration, 26th ICRC, Salt Lake City, USA, 2 (1999) 384. L. Scarsi et al. - EUSO Team, Proc. 27th ICRC, Hamburg, Germany, 2 (2001) 839

[98] R.A. Antonov et al., Proc. 27th ICRC, Hamburg, Germany, 2 (2001) 828 